\title{
Análisis complementario entre parámetros instrumentales y macrosísmicos: terremoto de mayo 24, 2008 en Quetame, Colombia
}

\author{
Elkin de Jesús Salcedo-Hurtado ${ }^{1 *}$; Augusto Antonio Gómez-Capera² ${ }^{\mathbb{D}}$; Héctor Mora-Páez ${ }^{3}$
}

Forma de citar: Salcedo-Hurtado, E.DeJ.; Gómez-Capera, A.A.; Mora-Páez, H. (2021). Análisis complementario entre parámetros instrumentales y macrosísmicos: terremoto de mayo 24, 2008 en Quetame, Colombia. Boletín de Geología, 43(2), 163-184. https://doi.org/10.18273/revbol.v43n2-2021009

\begin{abstract}
Resumen
El terremoto ocurrido el 24 de mayo de 2008 en el municipio de Quetame (Colombia), es un evento reciente detectado por la Red Sismológica Nacional de Colombia (RSNC) y la Red Sismológica Mundial, que reportaron parámetros como: epicentro, profundidad, magnitud, momento sísmico escalar y mecanismo focal, entre otros. Basado en los daños y efectos causados, INGEOMINAS (Servicio Geológico Colombiano) evaluó el campo macrosísmico elaborando el mapa de intensidades con datos en diversas poblaciones de los departamentos de Cundinamarca, Tolima y Meta, principalmente. Tomando como base esta información macrosísmica, en el presente trabajo se aplican algunos métodos de análisis macrosísmico para obtener los correspondientes parámetros focales. Los resultados muestran fuertes correlaciones o similitudes entre los parámetros

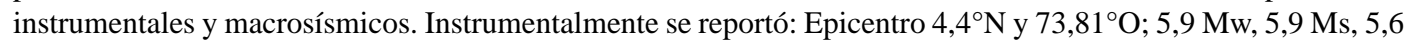
$\mathrm{mb}, 5,7 \mathrm{M}_{\mathrm{L}} ; \mathrm{h}=14,7 \mathrm{~km}, \mathrm{M}_{0}=7,95 * 10^{24}$ dinas-cm. Mientras que macrosísmicamente se obtuvo: Epicentro 4,34 $\mathrm{N}$ y 73,86 ${ }^{\circ}$; 5,94 Mw, 5,5 Ms, 5,6 $\pm 0,2 \mathrm{mb}, 5,63 \mathrm{M}_{\mathrm{L}} ; \mathrm{h}_{\mathrm{n}}=12,1 \mathrm{~km}, \mathrm{~h}_{1}=2,23 \mathrm{~km} ; \mathrm{E}=4,57 * 10^{20}$ ergios, $\mathrm{M}_{0}=9,14^{*} 10^{24}$ dinas-cm; también se determinaron los parámetros de las dimensiones del foco sísmico y la deformación sismotectónica expresada por el flujo sísmico de la masa rocosa. Los procedimientos aplicados muestran el carácter de complementariedad entre los datos instrumentales y macrosísmicos.
\end{abstract}

Palabras clave: Terremoto; Parámetros focales; Instrumentales; Macrosísmicos; Complementariedad.

\section{Complementary analysis between instrumental and macrosismic parameters: earthquake may 24, 2008 in Quetame, Colombia}

\begin{abstract}
The earthquake of May 24th, 2008, occurred in the municipality of Quetame (Colombia), is a recent event detected by the RSNC and the World Seismological Network, which reported their seismological parameters: epicenter, depth, magnitude, scalar seismic moment and focal mechanism, among others. Based on the damages and effects caused, INGEOMINAS (Colombian Geological Survey) evaluated the macroseismic field, preparing the map of intensities with data in various populations of the departments of Cundinamarca, Tolima and Meta, mainly. Based on this macroseismic information, in this work some macroseismic analysis methods are applied to obtain the corresponding focal parameters. The results show strong correlations or similarities between instrumental and macroseismic parameters. Instrumentally reported: Epicenter $4.4^{\circ} \mathrm{N}$ and $73.81^{\circ} \mathrm{W}$; $\mathrm{h}=14.7 \mathrm{~km}, 5.9 \mathrm{Mw}, 5.9 \mathrm{Ms}, 5.6 \mathrm{mb}, 5.7 \mathrm{M}_{\mathrm{L}} ; \mathrm{M}_{0}=7.95 * 10^{24}$ dynes-cm. While macroseismically it was obtained:

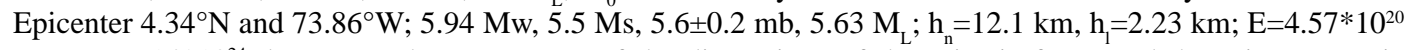
erg, $\mathrm{M}_{0}=9.14 * 10^{24}$ dynes-cm; the parameters of the dimensions of the seismic focus and the seismotectonic deformation expressed by the seismic flow of the rock mass were also determined. The applied procedures show the character of complementarity between instrumental and macroseismic data.
\end{abstract}

Keywords: Earthquake; Focal parameters; Instrumentals; Macroseismics; Complementarity.

\footnotetext{
${ }^{1}$ Grupo Georiesgos, Observatorio Sismológico y Geofísico del Suroccidente Colombiano, Departamento de Geografía, Universidad del Valle, Cali, Colombia (*) elkin.salcedo@correounivalle.edu.co

${ }^{2}$ Istituto Nazionale di Geofisica e Vulcanologia, sezione di Milano-Pavia, Milan, Italy. antonio.gomez@ingv.it

${ }^{3}$ Servicio Geológico Colombiano, Bogotá, Colombia. hmora@sgc.gov.co
} 


\section{Introducción}

Uno de los principales inconvenientes que se presenta para la valoración de la amenaza y el riesgo sísmico es la no completitud y falta de homogeneidad de los catálogos sismológicos (Stucchi, 1994), especialmente en regiones sísmicamente activas donde es notoria la escasez de instrumentación sismológica que realice el monitoreo de las fuentes sismogénicas y la detección confiable de los eventos sísmicos recientes y, por tanto, el periodo de registro de los datos instrumentales es muy corto.

La posible solución a este problema puede estar en ampliar el conocimiento de los terremotos históricos que, en ocasiones, resultan ser los más grandes en cuanto a la cantidad de energía sísmica liberada. Es decir, que se requiere de la valoración macrosísmica de los terremotos ocurridos en periodos preinstrumentales, lo cual es factible a partir de los estudios de sismicidad histórica, que propenden por el registro y descripción cualitativa de los efectos macrosísmicos de los terremotos ocurridos en el pasado que, al ser interpretados apropiadamente, permiten determinar los principales parámetros como el tiempo de ocurrencia, la localización epicentral, la intensidad y, en los casos posibles, la magnitud macrosísmica equivalente y la profundidad del foco. Existen modelos teóricos o empíricos que proponen el uso de información macrosísmica, en este caso de la intensidad sísmica, para valorar diversos parámetros focales de terremotos históricos (Blake, 1941; Shebalin, 1968; Bakun y Wentworth, 1997; Bakun, 2006), que también pueden ser aplicados sin restricción a terremotos ocurridos recientemente, cuyos parámetros pueden ser confrontados o complementados con los datos instrumentales reportados por las agencias sismológicas.

Es decir, que el análisis e interpretación del campo macrosísmico conduce a la determinación de diversos parámetros macrosísmicos que pueden complementarse con los definidos instrumentalmente, permitiendo la calibración entre datos macrosísmicos e instrumentales de terremotos recientes y con ello lograr ampliar la información de los catálogos sismológicos incluyendo datos para extensos periodos históricos.

En el presente trabajo se escoge el sismo del 24 de mayo de 2008 ocurrido en Colombia, con el fin de evaluar los parámetros macrosísmicos a partir de los datos reportados por INGEOMINAS (Sarabia y
Cifuentes, 2009), que realizó visitas de campo para levantar información, por un lado, para buscar la correlación del evento con alguna fuente sismogénica de la región, y, por otro lado, valorar la intensidad en la escala macrosísmicas europea (EMS-98) (Grünthal, 1998) y Mercalli Modificada (Bolt, 2003). El sismo fue localizado en las coordenadas $4,39^{\circ} \mathrm{N}$ y $73,81^{\circ} \mathrm{O}$ a 8,6 $\mathrm{km}$ al norte de la cabecera municipal del municipio de Quetame en el departamento de Cundinamarca, en una zona que corresponde a rocas del Cretácico Inferior, pudiendo ser asociado con la falla Naranjal (Figura 1).

Los parámetros instrumentales fueron reportados por la Red Sismológica Nacional de Colombia y la Red Sismológica Mundial a través de los catálogos emitidos por el Centro Sismológico Internacional (ISC por sus siglas en inglés) y por la Universidad de Harvard en el proyecto Global Centroid Moment Tensor (GCMT).

Usando la información sobre las intensidades propuestas por Sarabia y Cifuentes (2009) y aplicando los modelos macrosísmicos de Bakun y Wentworth (1997) y Shebalin (1974) y otras relaciones empíricas (Gutenberg y Richter, 1942; Kanamori, 1977, 1983; Bommer, 1994; Kostrov, 1974), se calculan los parámetros macrosísmicos del terremoto del 24 de mayo de 2008, equivalentes a los reportados instrumentalmente, obteniéndose una fuerte correlación entre ellos, mostrando la complementariedad entre los datos macrosísmicos e instrumentales. Esto permite sugerir el uso de estos modelos macrosísmicos para evaluar confiablemente los parámetros focales de los terremotos históricos para enriquecer los catálogos sismológicos y aportar en la solución del problema de la amenaza y riesgo sísmico local y regional.

\section{Marco teórico}

\section{Complementariedad de parámetros instrumentales y macrosísmicos de terremotos}

En las zonas o regiones de gran actividad sísmica, bien sea por la frecuencia de los terremotos o por la severidad de los mismos, es lógico plantear que cualquier medida de toma de decisión relacionada con la planeación del desarrollo y la planificación territorial regional o urbana, debe basarse en el conocimiento de las condiciones de los riesgos naturales que puedan presentarse. Para el caso particular de los terremotos, dada la limitante metodológica que aún se tiene para su predicción, dicho conocimiento se fundamenta en los denominados estudios de zonificación sísmica. 

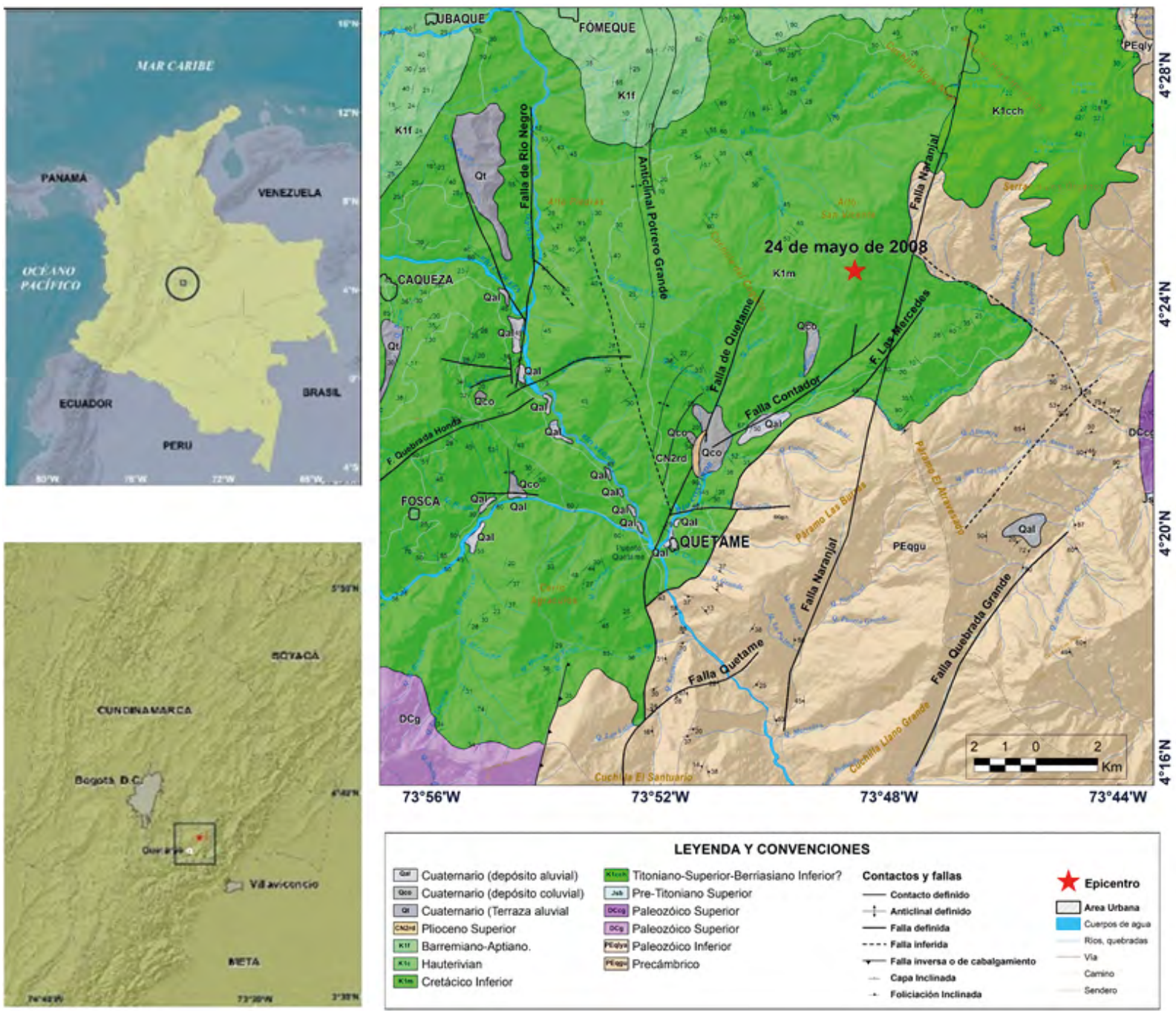

Figura 1. Esquema Geológico de la zona epicentral del terremoto de Quetame del 24 de mayo de 2008 (información tectónica a partir de Cortés y De La Espriella, 1990; Patiño et al., 2011).

La zonificación sísmica se entiende como la determinación y distribución espacial de algunas propiedades específicas de los terremotos que se evalúan con el propósito de proporcionar información suficiente para la toma de decisiones en torno a la planificación territorial y del desarrollo en una región determinada, teniendo como resultado un mapa que muestra una cantidad o cantidades relacionadas con la frecuencia e intensidad esperadas de la sacudida que pueden provocar los sismos futuros en las cercanías del lugar que se evalúe (Karnik y Algermissen, 1980). Para el análisis de la zonificación sísmica han sido considerados los enfoques determinístico y probabilístico, ligados respectivamente a la consideración del fenómeno sísmico como un proceso, probabilista o determinista (Reiter, 1990).
Desde el punto de vista del método determinista, la solución del problema de la amenaza implica que la ocurrencia de terremotos en el futuro se comportará de manera similar a lo ocurrido históricamente, es decir, que la magnitud o intensidad de un terremoto en el futuro no será superior a la máxima observada en el lugar determinado. Así, para la aplicación de este método es esencial contar con catálogos completos en el sentido que incluyan los terremotos más grandes posibles en la zona, y que la asignación de los correspondientes parámetros, en particular la intensidad máxima, sea correcta.

Por su parte, el método probabilista estima las tasas de sismicidad de una región dada en función de los datos de terremotos pasados; sin embargo, esto también 
requiere un catálogo completo de todos los terremotos significativos durante un extenso período de tiempo. Cabe señalar que los catálogos basados en registros instrumentales están disponibles en el mejor de los casos desde alrededor de 1900, y solo son confiables después de 1960 pero, aunque las fechas y los epicentros aproximados son probablemente confiables, es difícil obtener magnitudes y mecanismos focales confiables (Mulargia et al., 2017).

La falta de completitud de los catálogos, debido al corto periodo de registro instrumental o al bajo número y sensibilidad de las estaciones sismológicas, genera huecos o "gaps" en el número de terremotos de diversos rangos de magnitudes, de tal manera que el modelo de frecuencia de ocurrencia de Gutenberg-Richter pierde su linealidad dejando como consecuencia que para los cálculos probabilistas se requiera aplicar mecanismos de corrección, homogeneización o completitud de catálogos (Stepp, 1972; Cornell, 1986; Martín-Martín, 1989). El problema de no conocer todos los terremotos ocurridos en la zona para la aplicación del método probabilista obliga a que se debe modelar la ocurrencia de sismos en el tiempo y el espacio. Para ello MartínMartín (1989) plantea que lo usual es recurrir a modelos paramétricos, en los que la sismicidad se representa por un pequeño número de parámetros comparado con el número de terremotos.

Sin embargo, los catálogos paramétricos no son datos primarios puesto que éstos, a su vez, son los resultados de la elaboración realizada sobre otros datos (Stucchi, 1994). Por ejemplo, al comparar la naturaleza de los datos instrumentales y macrosísmicos, se puede notar que, en el caso de los datos instrumentales, dicha elaboración es bien conocida a partir de las formas de ondas, que son los respectivos datos primarios; similarmente, para el caso macrosísmico, los datos primarios son registros históricos o cuestionarios macrosísmicos, cuyo procesamiento se realiza mediante cuatro pasos básicos (Stucchi, 1994), como muestra en la Figura 2.

a. Nivel 1A - se investigan las fuentes históricas: los registros de terremotos (observaciones históricas) se encuentran y se ubican en el tiempo y el espacio, siendo equivalente a la localización y descripción de las características técnicas de las estaciones sismológicas para los datos instrumentales.

b. Nivel 1B - el investigador decide qué registros pertenecen al mismo evento y "selecciona" un terremoto: se evalúa el tiempo $T_{0}$ de ocurrencia del terremoto y se determinan los daños y efectos en diversos lugares, equivale a la lectura de las formas de ondas en el caso instrumental.

c. Nivel 2 - los registros de terremotos se interpretan en términos de intensidad macrosísmica, produciendo datos de puntos de intensidad (observaciones macrosísmicas); equivalente a la lectura de fases determinando las amplitudes $\mathrm{y}$ periodos en las formas de ondas registradas instrumentalmente.

d. Nivel 3 - se determinan los parámetros focales $\left(\lambda_{0}, \varphi_{0}, h_{0}, I_{0}, M_{0}, M w\right.$, etc.) que se evalúan de acuerdo con algunas reglas, y con los registros se compilan los catálogos; los mismos parámetros son determinados instrumentalmente.

Según Stucchi (1994), la diferencia real entre los datos macrosísmicos e instrumentales es que, por un lado, las formas de onda (Nivel 1A) son más fáciles de obtener $\mathrm{y}$, por otro lado, que los procedimientos mediante los cuales se determinan los datos instrumentales (desde el Nivel 1B hasta el Nivel 3), son conocidos y estandarizados, al menos en cierta medida: registros instrumentales del catálogo. Por lo tanto, son comparables, y la fiabilidad puede ser evaluada. Por el contrario, en el caso de los datos macrosísmicos, estas acciones no se realizan de acuerdo con procedimientos estandarizados. En tales circunstancias, los registros de los catálogos correspondientes a diferentes conjuntos de datos y procedimientos no se pueden comparar fácilmente.

Por consiguiente, a los resultados de los estudios de sismicidad histórica se les pueden aplicar relaciones matemáticas apropiadas (modelos macrosísmicos empíricos) donde a partir de la distribución espacial de las intensidades se obtienen parámetros focales similares a los deducidos instrumentalmente, como magnitud, localización epicentral, profundidad focal y tamaño de falla, entre otros. Así, pueden obtenerse relaciones que permiten deducir algún parámetro a partir del conocimiento de otro; sin embargo, dichas relaciones deben calibrarse primero con sismos recientes de los que se disponga tanto de resultados instrumentales como de distribuciones de intensidades (Izquierdo-Álvarez, 1999; Gómez-Capera et al., 2009, 2013; Storchak et al., 2012, 2013). Este procedimiento muestra el carácter de complementariedad entre los parámetros instrumentales y macrosísmicos referentes a un terremoto dado, brindando la posibilidad de lograr un mejor conocimiento de los terremotos en una región dada y mayor confiabilidad en los resultados de los estudios de amenaza y riesgo sísmico que se alimentan con dichos datos. 


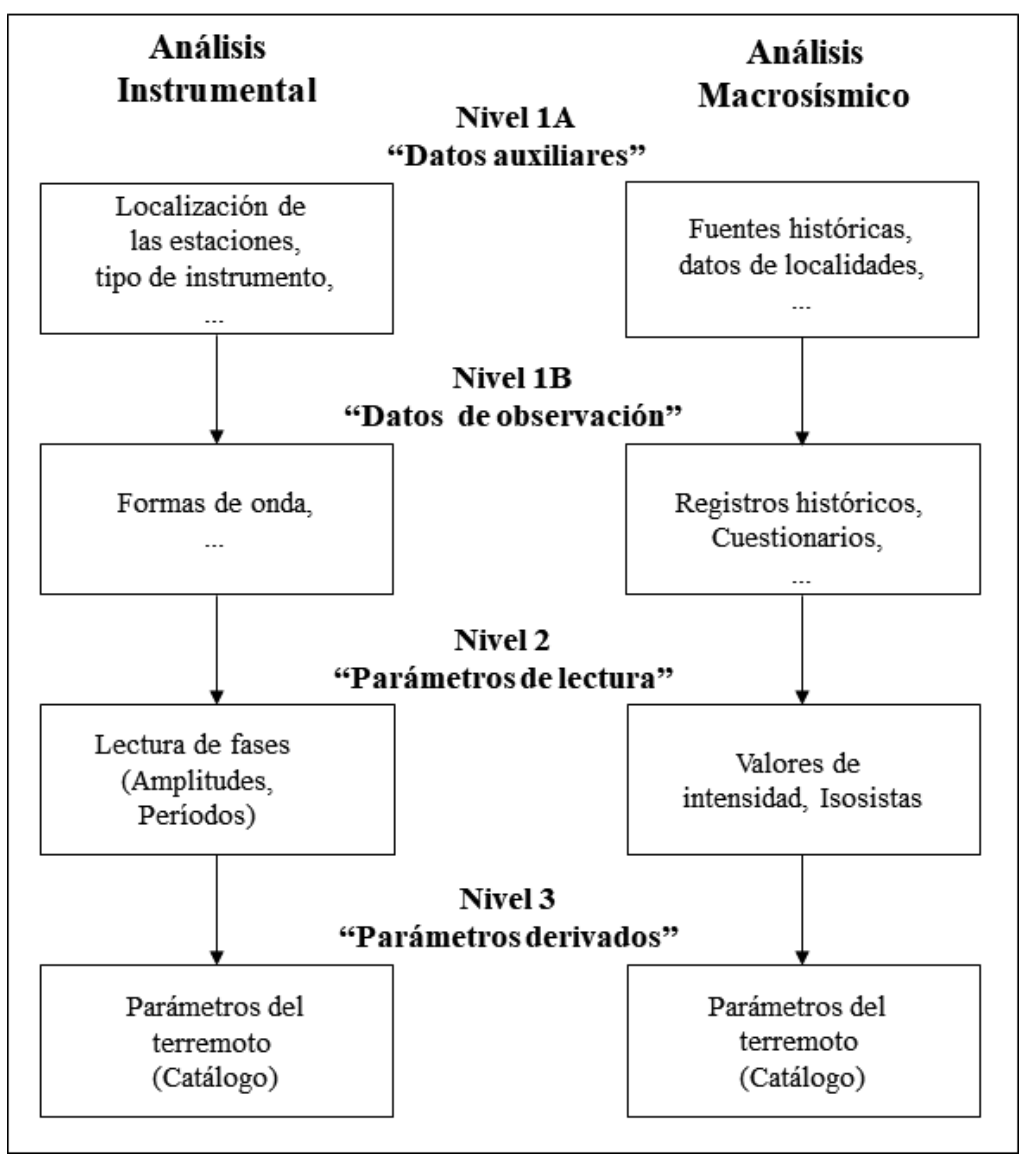

Figura 2. Niveles de procesamientos de datos instrumentales y macrosísmicos (modificado de Stucchi, 1994).

\section{Parámetros instrumentales de los terremotos}

Un terremoto ocurre por la liberación repentina de la energía de deformación acumulada en una falla geológica, que ante la ruptura parcialmente se transforma en ondas elásticas. Esta liberación de energía produce en la Tierra ondas internas o de cuerpo (longitudinales u ondas $\mathrm{P}$ y transversales u ondas S) y ondas superficiales (Rayleigh y Love). La lectura de las diferentes fases de las ondas sísmicas permite obtener los parámetros instrumentales de los terremotos, siendo la magnitud la principal medida del tamaño del terremoto, que puede ser valorada según la fase y el tipo de ondas que se examine (Lay y Wallace, 1995; Udías y Mezcua, 1997; Bormann et al., 2002; Bolt, 2003; Stein y Wysession, 2003). Así, se destacan los siguientes parámetros:

Magnitud local (Richter) $-\mathrm{M}_{\mathrm{L}}$ : Richter propuso esta magnitud tomando sismos de California, asumiendo que el tamaño relativo de los terremotos es calculado por comparación con un sismo de referencia, estableciendo que (Lay y Wallace, 1995; Udías y Mezcua, 1997):

$$
M_{L}=\log A-\log A_{0}
$$

Magnitud de ondas superficiales - Ms: esta escala se basa en la amplitud máxima producida por las ondas superficiales Rayleigh con período en el rango de 18 a 22 segundos. Se estima con la ecuación (Udías y Mezcua, 1997; Stein y Wysession, 2003):

$$
M s=\log \frac{A}{T}+1,66 \log \Delta+3,3
$$

Magnitud de ondas de cuerpo - mb: se basa en la amplitud de ondas de cuerpo con período cercano a 1,0 segundo, para su determinación se utiliza la siguiente expresión (Lay y Wallace, 1995; Udías y Mezcua, 1997; Stein y Wysession, 2003):

$$
m b=\log (A / T)+\sigma(\Delta)
$$


Magnitud Momento - Mw: la ecuación que relaciona el momento sísmico con la magnitud $\mathrm{Mw}$ fue introducida por Kanamori (1977). Es una escala más apropiada para medir el tamaño de los terremotos puesto que no utiliza una fase sísmica en particular, y tiene la forma:

$$
M w=\frac{2}{3}-\log M_{0}-10,7
$$

Donde $\mathrm{M}_{0}$ es el momento sísmico, medido en Nm (SI) o dinas-cm (cgs), que corresponde al torque del par de fuerza equivalente, y es utilizado para medir el tamaño de un terremoto (Stein y Wysession, 2003) en el sentido que representa el producto del desplazamiento relativo de la fractura por el área y el coeficiente de rigidez del medio en que se ha producido (Udías y Mezcua, 1997), y se expresa como:

$$
M_{0}=\mu \mathrm{DS}
$$

Siendo $\mu$ la rigidez de la roca, en dinas $/ \mathrm{cm}^{2}$, D es el desplazamiento promedio de la falla (en $\mathrm{cm}$ ) y S es el área del segmento que sufrió la ruptura expresada en $\mathrm{cm}^{2}$.

La Magnitud Mw es válida para todo el rango de valores, mientras que las demás se saturan, es decir que no proporcionan datos confiables a partir de cierto valor; en especial, esta escala da valores más precisos para magnitudes grandes, por encima de 8,0, que la escala de Ms (Kanamori, 1977; Hanks y Kanamori, 1979; Udías y Mezcua, 1997).

Energía sísmica: cuando se produce un terremoto causado por la ruptura a lo largo de una falla geológica, gran parte de la energía que se ha acumulado para la deformación de la roca se disipa en forma de calor; mientras que una parte menor es irradiada en forma de ondas elásticas, que producen las ondas sísmicas. Gutenberg y Richter (1942) desarrollaron una expresión que relaciona la energía liberada con la magnitud, dada de la siguiente forma (Kanamori, 1977; Hanks y Kanamori, 1979):

$$
\log E s=1,5 M s+11,8
$$

\section{Parámetros macrosísmico)}

(campo

En general, los efectos causados por un terremoto se distribuyen en la superficie terrestre dependiendo de tres factores principalmente: 1) el campo de emisión, que define las características de la fuente sísmica, representada en el tipo y mecanismo de la ruptura asociada con el terremoto; 2) el campo de transmisión, definido por las propiedades físicas del medio en el interior de la tierra, que condicionan la propagación de las ondas sísmicas, y 3) el campo de recepción que, por un lado, depende de las propiedades de los suelos que pueden atenuar o amplificar las ondas sísmicas, $y$, por otro lado, de la vulnerabilidad de los elementos expuestos, como objetos, personas y estructuras (infraestructura). El campo de recepción condiciona los daños y efectos perceptibles causados por el terremoto en un lugar determinado, configurándose con ello el llamado campo macrosísmico (Shebalin, 1968; Salcedo-Hurtado, 2002).

El campo macrosísmico se define como la región geográfica en la superficie de la tierra, alrededor del epicentro en la que interactúa la fuerza del terremoto con los elementos que ocupan dicho espacio, de tal manera que en ella el sismo puede ser percibido y en algunos casos producir daños y efectos considerables. Esto implica que el campo macrosísmico no es medible directamente, sino lo que es observable es su efecto sobre algunos elementos expuestos que tienen algún grado de vulnerabilidad como: objetos, personas, construcciones y el suelo o terreno propiamente dicho.

Los efectos sobre los elementos expuestos se determinan por un valor de intensidad que se evalúa a partir del uso de una escala macrosísmica como: Mercalli Modificada, MSK-64 (Bolt, 2003), Escala Macrosísmica Europea (EMS-98), entre otras. Matemáticamente, el campo macrosísmico se expresa mediante una fórmula que relaciona el decaimiento de la intensidad con el aumento de la distancia epicentral, así:

$$
I_{i}=I_{0}-f\left(r_{i}\right)
$$

Donde $\mathrm{I}_{\mathrm{i}}$ es la intensidad medida en un punto cualquiera de la superficie terrestre, $\mathrm{I}_{0}$ representa la intensidad en el epicentro y $\mathrm{r}_{\mathrm{i}}$ la distancia hipocentral que se expresa como:

$$
r i=\sqrt{x_{\mathrm{i}}^{2}+h^{2}}
$$

$\mathrm{x}_{\mathrm{i}}$ corresponde a la distancia epicentral y h representa la profundidad del foco del terremoto.

Existen diversos modelos teóricos que proponen el uso de la información macrosísmica, es decir, sobre la 
distribución de los efectos causados por un terremoto, que permiten valorar diferentes parámetros focales como la magnitud y la profundidad, entre otros. Uno de los primeros métodos propuestos es el de Kövesligethy (1906, citado en Zsìros, 1995), el cual fue modificado por Blake (1941). Otros modelos más recientes son los propuestos por Shebalin (1968), Bakun y Wentworth (1997) y Bakun (2006).

\section{Efecto de extensión del foco del terremoto}

Algunos modelos sobre las dimensiones del foco de un terremoto suponen que la dislocación que se produce durante el sismo se extiende a lo largo de una superficie $\mathrm{S}$ determinada, siendo necesario especificar la geometría de la superficie de la fractura. Los modelos más sencillos son el de una fractura rectangular y el de una fractura circular, propuestos por Haskell (1964) y Brune (1970), respectivamente. De acuerdo con Madariaga (1985, 1994), el modelo de la fractura circular puede ser considerado como un modelo intermedio entre los modelos cinemáticos y los dinámicos. Un caso particular de un modelo cinemático, es el propuesto por Shebalin (1974), mediante el cual el foco de un terremoto puede considerarse como un punto para toda distancia hipocentral, expresada en la ecuación 8.

Savage (1966) propuso un modelo sísmico en el que considera una fractura elíptica generada por un terremoto, que contiene un efecto de comienzo o nucleación y la correspondiente parada o finalización (Udías, 1994). De acuerdo con este modelo, la nucleación se origina en uno de los focos que, al prolongarse hacia la superficie se detiene cuando llega al borde de la elipse. Este corresponde al hipocentro del terremoto, evaluado instrumentalmente, cuya profundidad es denominada profundidad normal (Shebalin, 1968, 1971, 1974), definida como:

$$
\log h_{n}=0,3 M-0,7
$$

donde $h_{n}$ expresada en kilómetros es la profundidad normal del foco y M la magnitud del terremoto.

De acuerdo con Shebalin (1968, 1971), el modelo de comienzo y finalización de la fractura elíptica causada por el terremoto, expresa la extensión física horizontal y vertical del foco sísmico; de tal manera que éste corresponde a un modelo de sucesión de subfocos, que se propagan oblicuamente como resultado de la extensión horizontal y vertical de la ruptura; es decir, que entre los focos de iniciación y terminación de la fractura elíptica, existe una sucesión finita de subfocos (cadenas y barreras), a través de los cuales progresivamente se va transmitiendo la radiación de la energía elástica (Shebalin, 1974).

Extensión horizontal del foco. La extensión horizontal del foco sísmico, representada como $l_{x}$, corresponde a la cadena de subfocos que se propagan horizontalmente. $\mathrm{Al}$ representar el campo macrosísmico del terremoto en un mapa de isosistas, se puede suponer que la forma de la primera isosista hereda la extensión horizontal del foco local, para lo cual se asume que se desprecia la absorción anisotrópica en las pequeñas distancias; así, de manera general, la extensión horizontal se define como (Shebalin, 1991):

$$
l_{X}=d_{1 \max }-d_{1 \min }
$$

Donde $\mathrm{d}_{1 \max } \mathrm{y} \mathrm{d}_{1 \min }$ representan, respectivamente, el diámetro mayor y menor de la isosista 1 con mayor valor de intensidad (Shebalin, 1991; Marín-Arias et al., 2006).

Para el caso de un terremoto de gran magnitud, que manifieste efectos locales y estos coincidan con el centro de las isosistas, es posible que estos efectos se relacionen con la primera isosista, que a su vez corresponde al efecto local de la extensión vertical del foco. En este caso, claramente, la extensión horizontal del foco no se manifestaría en la primera isosista sino en la subsiguiente, de tal manera que $l_{x}$ está representado por el promedio de las 2 primeras isosistas (Shebalin, 1991).

La extensión horizontal del foco manifiesta uno de los factores por los cuales se presenta la no coincidencia de los epicentros instrumental y macrosísmico de un terremoto. Para terremotos donde es evidente la aparición de una gran extensión horizontal del foco, el epicentro macrosísmico se toma como el baricentro de la primera isosista.

Extensión vertical del foco y Profundidad focal. La extensión vertical del foco es un efecto macrosísmico que ocurre debido a que, por causa de la rigidez del medio, la parte superior del foco del terremoto, en comparación con la parte inferior, se enriquece de subfuentes (rupturas secundarias), creándose en ese lugar un exceso de fuentes de efectos macrosísmicos; de tal manera que dicha extensión, de acuerdo con Shebalin (1974), cumple con la siguiente condición: 


$$
\log l_{z}=0,3 M-0,8
$$

Donde $\mathrm{l}_{\mathrm{z}}$ es la extensión vertical del foco sísmico y M es la magnitud del terremoto.

Así, el modelo de un foco sísmico con gran extensión vertical, que se asume como una ruptura elíptica, se representa por dos fuentes, donde una de ellas corresponde al centro geométrico de la parte inferior del foco y su profundidad designa la profundidad focal normal $h_{n}$; la segunda fuente, se relaciona con el centro de la parte superior del foco, correspondiente a la profundidad focal local $h_{\text {. }}$. Por tanto, la extensión vertical del foco queda definida por la relación entre $h_{n}$ y $h_{1}$ de la siguiente manera (Shebalin, 1974):

$$
l_{z}=1,5\left(h_{n}-h_{z}\right)
$$

\section{Deformación sismotectónica}

El análisis de la deformación sismotectónica involucra el concepto de flujo sísmico de la masa rocosa propuesto inicialmente por Riznichenko (1965) cuyo significado físico, en términos de la mecánica del medio continuo, fue establecido por Kostrov (1974). La deformación sismotectónica es calculada a partir de la siguiente ecuación (Brune, 1968; Aki y Richards, 1980; Riznichenko, 1976; Riznichenko y Jibladze, 1976; Kostrov y Das, 1988):

$$
\dot{\varepsilon}_{i j}=\frac{1}{2 \mu V T}\left[M_{i j}\right]
$$

Donde $\dot{\varepsilon}_{i j}$ es el tensor de deformación, $\mathrm{M}_{\mathrm{ij}}$ representa las componentes del tensor de momento sísmico, $\mathrm{V}$ es el volumen de la fractura o el que corresponde a la deformación debido a la actividad o proceso sísmico, $\mathrm{T}$ es el tiempo de la deformación sísmica, correspondiente al período en que se desarrolla la fractura y relajación de los esfuerzos dentro de su volumen, el cual es concordante con el tiempo de duración del evento principal y sus réplicas, $\mu$ es el módulo de rigidez del medio o ambiente sismotectónico (Kanamori y Astiz, 1985; Heuret et al., 2011).

La velocidad media de deformación dentro del volumen se determina a partir de la orientación del campo de esfuerzos (Guzmán-Speziale, 2001), usando la siguiente ecuación:

$$
V_{i j}=l_{i j} \dot{\varepsilon}_{i j}
$$

Donde $\mathrm{l}_{1}, \mathrm{l}_{2} \mathrm{y}_{3}$ son las dimensiones del volumen a lo largo de las direcciones $x, y$ y $z$, que en el sistema de coordenadas cartesianas se orientan de tal manera que $x$ está en la dirección norte (N), $y$ hacia el este (E) y z hacia el centro de la Tierra.

\section{Metodología}

En el presente trabajo se aplican los métodos macrosísmicos empíricos propuestos por Bakun y Wentworth (1997), en adelante citado como B\&W97, y Shebalin $(1968,1971,1974)$, para determinar los parámetros focales del terremoto del 24 de mayo de 2008, mostrando que los resultados obtenidos son compatibles con los determinados instrumentalmente por las diversas agencias que reportaron los respectivos datos. A continuación, en esta sección, se describen como operan estos modelos macrosísmicos y los datos utilizados.

\section{Método Bakun \& Wentworth (B\&W97)}

Bakun y Wentworth (1997) propusieron un método que permite la valoración del epicentro y la magnitud de terremotos a partir de datos macrosísmicos. Este método, ha sido utilizado exitosamente en diversas regiones del mundo como: California (Bakun, 2006), Ecuador (Beauval et al., 2010), Venezuela (Choy et al., 2010), Suiza (Álvarez-Rubio et al., 2012), Haiti (Bakun et al., 2012), Asia central (Bindi et al., 2013, 2014), sureste de Cuba (Gómez-Capera et al., 2013), Colombia (Gómez-Capera et al., 2020) y en estudios de incertidumbres como en Bakun et al. (2011). Particularmente, en Colombia este método ha sido aplicado por Salcedo-Hurtado y Gómez-Capera (2013) y Gómez-Capera et al. (2014), para evaluar los parámetros focales de los terremotos de 1743 y 1785, respectivamente. Tradicionalmente, el método B\&W97, para el modelo de atenuación, usa una función lineal que involucra la magnitud de momento y el log de la distancia epicentral, dada como:

$$
I_{i j}=a+b M w-c R_{i j}-d\left(\log _{10} R_{i j}\right)
$$

donde $a, b, c$ y $d$ son constantes calibradas de acuerdo a la región donde se hace el estudio. En particular $c$ corresponde al coeficiente de atenuación anaelástico y $d$ al coeficiente de atenuación geométrico (Howell y Schultz, 1975). Igualmente, describen el proceso para determinar la localización y la magnitud de un j-ésimo terremoto dado a partir del cómputo de $\mathrm{MI}_{\mathrm{ij}}^{\mathrm{k}}$ sobre una malla de puntos de posibles epicentros localizados en $x_{k}$ (latitud $_{k}$, longitud $_{k}$ ). Finalmente, la magnitud de intensidad es determinada considerando las raíces medias cuadráticas (rms[M] $]$ ) (Gómez-Capera et al., 2013). 


\section{Método Shebalin}

De acuerdo con Shebalin (1968), el foco de un terremoto puede considerarse puntual solo si éste se ubica en una distancia suficientemente lejana del punto de observación. La existencia del campo macrosísmico presupone la aparición de los efectos de extensión horizontal y vertical del foco, descritos anteriormente. En términos generales, el método Shebalin para la representación del campo macrosísmico, es expresado mediante la siguiente ecuación:

$$
I_{i}=a_{i}-\gamma \log x_{i}
$$

Donde, según la ecuación 7, $\mathrm{a}_{\mathrm{i}}=\mathrm{I}_{0} \mathrm{y} \gamma \log \mathrm{x}_{\mathrm{i}}=\mathrm{f}\left(\mathrm{x}_{\mathrm{i}}\right)$. La constante $\gamma$ es el coeficiente de atenuación geométrica, denominado también como coeficiente de BlakeShebalin (Shebalin, 1968).

\section{Datos utilizados}

Parámetros instrumentales. Los parámetros instrumentales (magnitud, y coordenadas hipocentrales) del terremoto del 24 de mayo de 2008 fueron reportados por la Red Sismológica Nacional de Colombia (RSNC), utilizando 21 estaciones sismológicas localizadas en todo el territorio nacional, y por la Red Sismológica mundial, cuyos datos sobre el tiempo de ocurrencia, localización del epicentro, profundidad focal, magnitud, momento sísmico escalar y mecanismo focal, han sido publicados en los catálogos del International Seismological Center (ISC), del proyecto Global Centroid Moment Tensor (GCMT) de la Universidad de Harvard.

Parámetros reportados por la RSNC: se reporta la ocurrencia del sismo a las 14:20 hora local, con epicentro en las coordenadas $4,399^{\circ} \mathrm{N}$ y $73,814^{\circ} \mathrm{O}$ a $8,6 \mathrm{~km}$ al norte de la cabecera municipal del municipio de Quetame en el departamento de Cundinamarca, profundidad focal superficial y Magnitud de Richter 5,7 $\mathrm{M}_{\mathrm{L}}$ (INGEOMINAS, 2008).

Parámetros reportados por ISC y GCMT: los catálogos del ISC (2008) y del proyecto GCMT (Global CMT Catalog, 2009), reportaron magnitudes $\mathrm{Mw}=5,9$, Ms=5,9 y mb=5,6; el momento sísmico escalar $\mathrm{M}_{0}=7,95 * 10^{24}$ dinas-cm y profundidad de $14,7 \mathrm{~km}$. Como ejemplo de los reportes dados, se muestra el correspondiente al GCMT (Tabla 1).

Tabla 1. Datos instrumentales del terremoto de Quetame (Colombia) del 24 de mayo de 2008, tomados del GCMT.

\begin{tabular}{|c|}
\hline $\begin{array}{l}\text { Global CMT Catalog: https://www.globalcmt.org. } \\
\text { 200805241920A COLOMBIA }\end{array}$ \\
\hline Date: $2008 / 5 / 24$ Centroid Time: 19:20:48.7 GMT \\
\hline Lat $=4.32 \quad$ Lon $=-73.79$ \\
\hline Depth $=14.7 \quad$ Half duration= 2.1 \\
\hline $\begin{array}{lllllll}\text { Centroid time minus hypocenter time: } & 6.2 \\
\text { Moment Tensor: }: \text { Expo }=24 & 0.695 & 3.860 & -4.560 & -1.050 & 0.665 & 6.640\end{array}$ \\
\hline$M w=5.9 \quad \mathrm{mb}=5.6 \quad M s=5.9 \quad$ Scalar Moment $=7.95 \mathrm{e}+24$ \\
\hline Fault plane: strike=196 dip $=82$ slip $=-179$ \\
\hline Fault plane: strike=106 \\
\hline
\end{tabular}

La solución del mecanismo focal muestra uno de los planos nodales con los siguientes parámetros: azimut $\varphi=106^{\circ}$, buzamiento $\delta=89^{\circ}$ y deslizamiento $\lambda=-8^{\circ}$ (http://www.globalcmt.org/CMTsearch.html), el cual es compatible con la información tectónica de la falla Naranjal (INGEOMINAS, 2008; Patiño et al., 2011) (ver Figura 1), que corresponde a una falla de desgarre lateral izquierdo con orientación de $\mathrm{N} 15^{\circ} \mathrm{E} / 90^{\circ}$ (Cortés y De La Espriella, 1990). De acuerdo con Mora et al. (2006), históricamente, esta falla solo ha sufrido una reactivación compresiva menor.
Parámetros macrosísmicos. Los datos macrosísmico (valores de intensidad) son tomados del estudio macrosísmico realizado por INGEOMINAS (actualmente Servicio Geológico Colombiano) (Sarabia y Cifuentes, 2009). Dicho estudio propone hipótesis de intensidades en la escala macrosísmica europea de 1998 (Grünthal, 1998) y Mercalli Modificada (MM) para localidades afectadas de los departamentos de Cundinamarca, Meta y Tolima en el territorio colombiano. Muchas de las poblaciones afectadas por este sismo también sufrieron daños con 
los terremotos del 18 de octubre de 1743 y 12 de julio de 1785, cuya zona pleistosísmica coincide con la del terremoto del 24 de mayo de 2008 (Salcedo-Hurtado y Castaño-Castaño, 2011; Gómez-Capera et al., 2014).

Intensidad macrosísmica: Sarabia y Cifuentes (2009) establecen que la población más afectada por este sismo fue el municipio de Quetame, donde muchas construcciones de tipo comercial y residencial resultaron averiadas y algunas colapsaron. La iglesia y varias casas tuvieron que ser derribadas; otros municipios afectados fueron Fosca y Fómeque en el departamento de Cundinamarca y El Calvario y San Juanito en el departamento de Meta, donde se averiaron varias casas, iglesias, escuelas, hospitales, siendo necesario demoler algunas de esas construcciones. En Cáqueza se presentaron fisuras y grietas leves en algunas construcciones del casco urbano, mientras que en la zona rural varias viviendas sufrieron daños, quedando algunas de ellas inhabitables. En Guayabetal y Chipaque, se registraron daños leves. En la Figura 3 se presenta el mapa de intensidades evaluadas en cada una de las poblaciones afectadas, en total se presentan 19 puntos de intensidad, que en la escala EMS-98, están entre III y VIII.

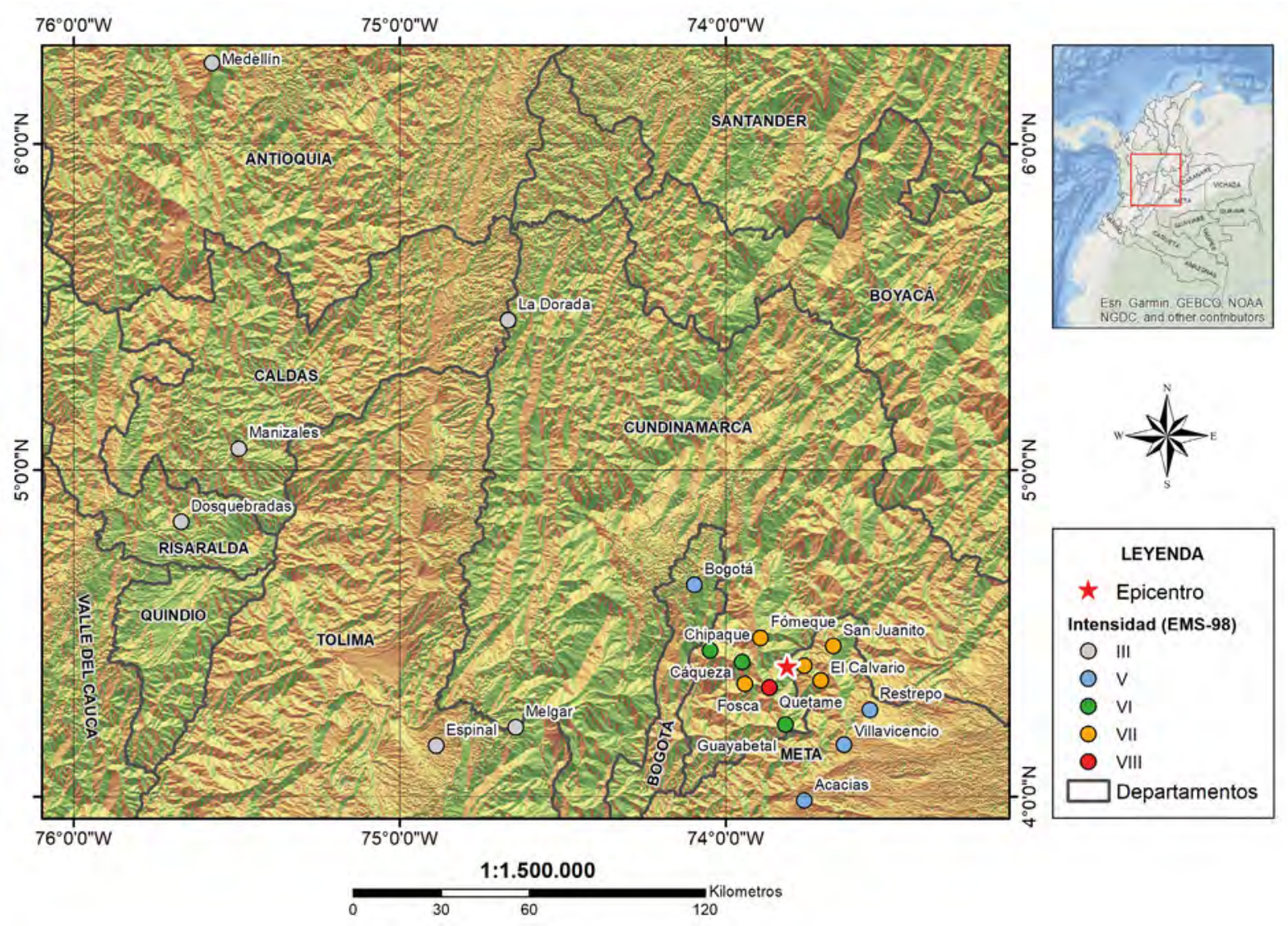

Figura 3. Mapa de intensidades macrosísmicas del terremoto del 24 de mayo de 2008 en cercanías del municipio de Quetame, Colombia (adaptada de Sarabia y Cifuentes, 2009).

De igual manera, Sarabia y Cifuentes (2009) apuntan que el sismo del 24 de mayo de 2008 se sintió fuertemente en Bogotá, Villavicencio, Acacias y Restrepo; señalan además que los mayores efectos inducidos por el sismo fueron los movimientos en masa (flujo, deslizamiento, desplome y desprendimiento) observados en Quetame,
Puente Quetame, Fómeque y El Calvario. Este mismo tipo de efectos también fueron observados durante los sismos del 18 de octubre de 1743 y 12 de julio de 1785 (Salcedo-Hurtado y Castaño-Castaño, 2011; SalcedoHurtado y Gómez-Capera, 2013). 


\section{Resultados}

\section{Aplicación del Método B\&W97}

Magnitud y epicentro macrosísmico. La magnitud y el epicentro macrosísmico del terremoto del 24 de mayo de 2008, son evaluados siguiendo el método B\&W97. Los datos macrosísmicos utilizados corresponden a los puntos de intensidad iguales o mayores que $\mathrm{V}$, publicados por el Servicio Geológico Colombiano (ver Figura 3). La intensidad máxima evaluada en la población de Quetame es 8 EMS98; en las localidades de El Calvario, Fosca, Fomeque, San Juanito, San Francisco se asigna intensidad 7; para las poblaciones de Cáqueza, Chipaque, Guayabal la intensidad es de 6; mientras que, para las ciudades de Villavicencio,
Bogotá, Acacías y Restrepo son asignadas intensidades de 5 EMS98.

Respecto a los modelos de atenuación de la intensidad macrosísmica, en el presente trabajo se usa el modelo GC\&SH2002 propuesto por Gómez-Capera y SalcedoHurtado (2002), y el de calibración del proyecto SARA, modelo SARA2017 (Gómez-Capera et al., 2017, 2020), ambos para el territorio colombiano.

En la Tabla 2 se presentan los parámetros instrumentales reportados por las agencias nacionales e internaciones, los datos macrosísmicos reportados por Sarabia y Cifuentes (2009) y los parámetros macrosísmicos calculados aplicando el método Bakun y Wentworth (1999) utilizando los modelos de atenuación GC\&SH2002 y SARA2017.

Tabla 2. Parámetros instrumentales vs macrosísmicos. RSNC = Red Sismológica Nacional de Colombia. $\mathrm{N}=$ número de puntos de intensidad macrosísmica. $\mathrm{I}_{\mathrm{x}}=$ intensidad macrosímica máxima en EMS98.

\begin{tabular}{|c|c|c|c|c|c|c|c|c|c|c|c|c|}
\hline \multicolumn{7}{|c|}{ Parámetros instrumentales } & \multicolumn{3}{|c|}{$\begin{array}{c}\text { Datos } \\
\text { macrosísmicos } \\
\text { intensidad } \\
\end{array}$} & \multicolumn{3}{|c|}{$\begin{array}{c}\text { Parámetros } \\
\text { macrosísmicos }\end{array}$} \\
\hline \multirow{2}{*}{ Fecha } & \multirow{2}{*}{ Lat. } & \multirow{2}{*}{ Long. } & \multirow{2}{*}{$\begin{array}{c}\mathbf{H} \\
(\mathbf{k m})\end{array}$} & \multirow{2}{*}{$\begin{array}{c}\text { Fuente } \\
\text { (hipocentro) }\end{array}$} & \multirow{2}{*}{$\mathbf{M}$} & \multirow{2}{*}{$\begin{array}{c}\text { Fuente } \\
\text { (M) }\end{array}$} & \multirow{2}{*}{ Fuente } & \multirow{2}{*}{$\mathbf{N}$} & \multirow{2}{*}{$\mathbf{I}_{\mathbf{x}}$} & \multicolumn{3}{|c|}{ Modelo GC\&SH2002 } \\
\hline & & & & & & & & & & Lat. & Long. & mb \\
\hline \multirow{4}{*}{ 2008.05.24 } & \multirow{4}{*}{4,40} & \multirow{4}{*}{$-73,81$} & \multirow{4}{*}{ Sup. } & \multirow{4}{*}{ RSNC } & 5,5 & \multirow{4}{*}{ GCMT } & \multirow{4}{*}{$\begin{array}{c}\text { Sarabia y } \\
\text { Cifuentes } \\
\text { (2009) }\end{array}$} & \multirow{4}{*}{21} & \multirow{4}{*}{8} & 4,34 & $-73,86$ & $5,6 \pm 0,2$ \\
\hline & & & & & $\mathrm{mb}$ & & & & & \multicolumn{3}{|c|}{ Modelo SARA2017 } \\
\hline & & & & & & & & & & Lat. & Long. & Mw \\
\hline & & & & & $\begin{array}{l}5,9 \\
\mathrm{Mw}\end{array}$ & & & & & 4,42 & $-73,81$ & $5,80 \pm 0,17$ \\
\hline
\end{tabular}

En la Figura 4A se muestra el resultado de la modelación matemática que evalúa el Centro de Intensidades CI a $9 \mathrm{~km}$ al sur-este del epicentro instrumental dentro del $50 \%$ y $95 \%$ de intervalos de confianza y a $42 \mathrm{~km}$ al sur-este de Bogotá; de tal manera que la magnitud

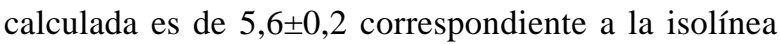
azul que intercepta el epicentro macrosísmico, siendo ésta una magnitud equivalente a mb usada en el modelo de atenuación GC\&SH2002; la incertidumbre media es de $\pm 0,2$ en el $67 \%( \pm 1 \sigma)$ del nivel de confianza y 0,3 en el $95 \%( \pm 2 \sigma)$ del nivel de confianza. La respectiva curva de atenuación se muestra en la Figura 5A.

La Figura 4B muestra el resultado de la modelación matemática que determina el CI en las coordenadas $4,42^{\circ}$ de latitud Norte y $73,81^{\circ}$ de longitud Oeste, calculando la magnitud $\mathrm{Mw}$ igual a 5,8 $\pm 0,17$, usada en el modelo SARA2017. La diferencia de 0,1 unidad de magnitud Mw respecto al reportado por el proyecto GCMT, puede estar asociada, por un lado, al hecho que la curva de atenuación tiende a anticipar los valores altos de intensidad para $x<25 \mathrm{~km}$, por efecto de la singularidad del modelo a distancias nulas es decir muy cercana de la fuente; por otro lado, a distancias $x>100$ $\mathrm{km}$ el modelo de atenuación tiende a sobreestimar la intensidad en relación a las intensidades observadas (Figura 5B).

Mediante el uso de las relaciones entre Mw y mb propuestas por Storchak et al. (2012, 2013), tiene validez la conversión del valor de magnitud (5,6 mb) obteniendo valores de magnitud momento de 5,7 Mw y 5,94 Mw. El valor de $\mathrm{Mw}=5,94$ es concordante con la magnitud Mw reportada por Global CMT. Las relaciones indicadas se expresan como:

$$
M w=\exp ^{(-4,66+0,86 m b)}+4,56 ; 4,5 \leq m b \leq 6,0
$$




$$
M w=1,38 m b-1,79
$$

La magnitud 5,8 $\mathrm{Mw}$ con error de 0,17 es un resultado directo en dicha unidad puesto que en el modelo SARA2017 el decaimiento de la intensidad macrosísmica ha sido calibrado con la magnitud Mw (Ecuación 15). La diferencia con la magnitud propuesta por el proyecto GCMT fue explicada arriba. Mientras que la magnitud 5,94 Mw, deducida con el modelo GC\&SH2002, se obtiene después de realizar la conversión con las ecuaciones propuestas por Storchak et al. (2012, 2013), tiene validez debido a que se trata de un evento que no superó la magnitud 6,0 por lo cual la magnitud mb no estaría saturada. Este valor es similar al propuesto por el GCMT, por tanto, será el que se utilice para el cálculo de los parámetros macrosísmicos subsiguientes.
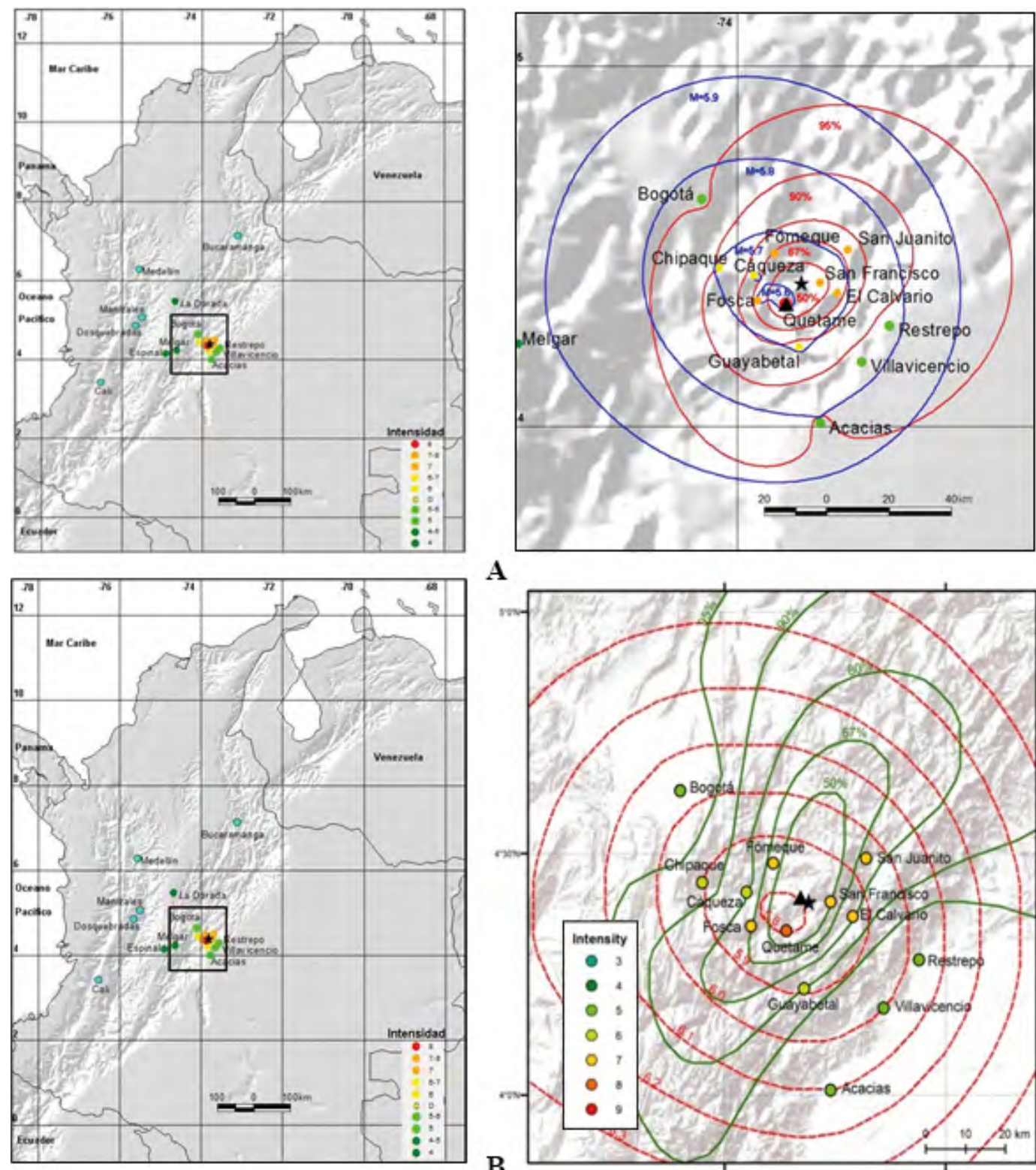

A

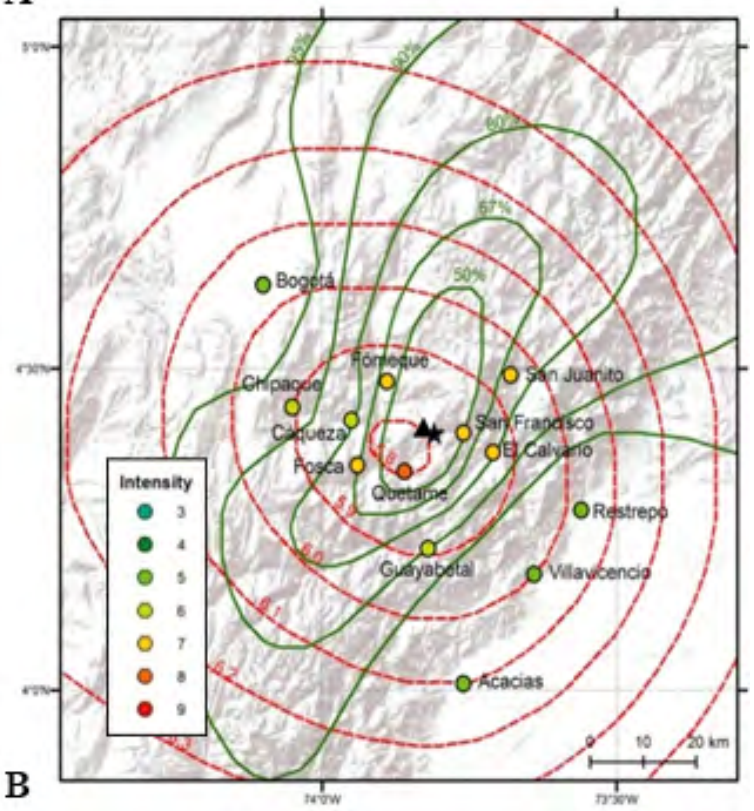

Figura 4. Representación gráfica de los parámetros macrosísmicos del terremoto del 2008.05.24 en Quetame. Puntos de intensidad macrosísmica tomados de Sarabia y Cifuentes (2009). Intensidad máxima en Quetame (I=8EMS98). Epicentro instrumental (estrella, 4,40N y 73,81O). A. Centro de intensidades adoptado como epicentro macrosísmico representado por el triángulo (4,34N y 73,860) propuesto en el presente estudio, magnitud instrumental 5,5 mb(Global CMT). Magnitud calculada 5,6 mb. Las isolíneas de color azul corresponden a los contornos de magnitud macrosísmica, y las de color rojo a los contornos de rms[M] para los intervalos de confianza. B. Centro de intensidades adoptado como epicentro macrosísmico representado por el triángulo (4,42N y 73,81O) propuesto en el presente estudio, magnitud instrumental 5,9 Mw(Global CMT). Magnitud calculada 5,8 $\pm 0,17$ Mw. 

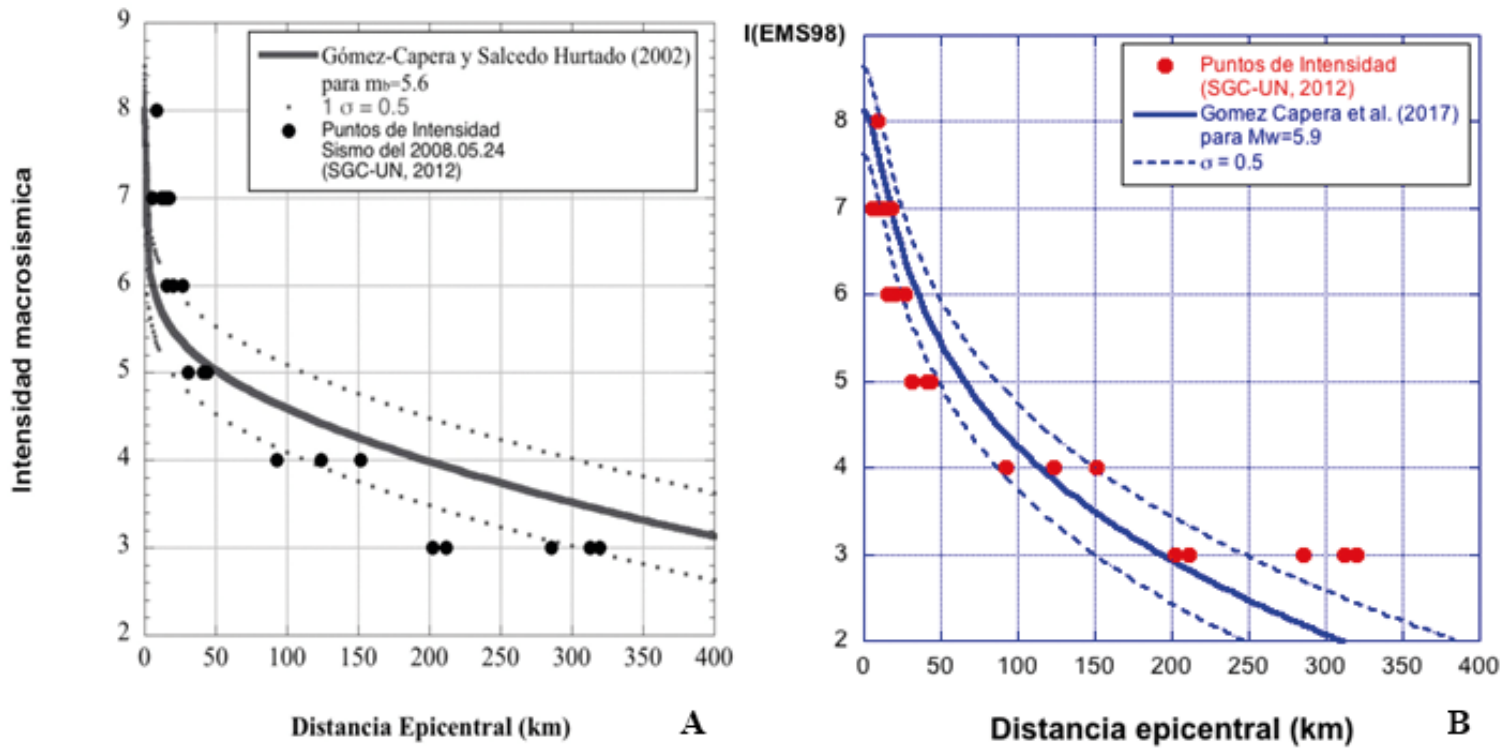

Figura 5. Intensidades macrosísmicas observadas del terremoto del 2008.05.24. A. Con 5,5 mb comparado con la curva de atenuación de GC\&SH2002 para una 5,6 mb (línea sólida). B. Con 5,9 Mw comparado con la curva de atenuación SARA2017 para una 5,8 $\pm 0,17$ Mw (línea sólida).

\section{Aplicación del Método Shebalín}

Dimensiones del foco. Teniendo en cuanta que, para el sismo del 24 de mayo de 2008 se ha calculado la magnitud macrosísmica equivalente a una magnitud $M w=5,94$, en el presente trabajo se obtienen los parámetros de las dimensiones del foco sísmico de la siguiente manera:

$\begin{array}{lc}\text { Extensión horizontal: } & l_{x}=22,8 \mathrm{~km} \\ \text { Extensión vertical: } & l_{z}=14,8 \mathrm{~km} \\ \text { Longitud del plano de ruptura: } & L=21,1 \mathrm{~km} \\ \text { Ancho del plano de ruptura: } & W=8,6 \mathrm{~km} \\ \text { Área de la superficie de ruptura: } & S_{o}=218,8 \mathrm{~km}^{2}\end{array}$

Se puede apreciar que los valores de $\mathrm{l}_{\mathrm{x}} \mathrm{y} \mathrm{L}$, en términos generales, son equivalentes. El valor de $\mathrm{S}_{0}$ es significativo al ser comparado con el resultado de $\mathrm{L} * \mathrm{~W}=181,5 \mathrm{~km}^{2}$. Estos parámetros son similares a los obtenidos por Mora-Páez et al. (2015).

Profundidad focal normal $\left(\mathrm{h}_{\mathrm{n}}\right)$ : considerando la aparición del efecto local causado por el sismo del 24 de mayo de 2008, y usando el criterio de Shebalin (1974), que denota el efecto macrosísmico de extensión vertical del foco, se obtiene que la profundidad focal normal es:

$$
h_{n}=12,1 \mathrm{~km} \text {. }
$$

Este valor es muy cercano a la profundidad reportada instrumentalmente por la Universidad de Harvard (CMT), cuyo valor es de $14,7 \mathrm{~km}$.

Profundidad focal local $\left(h_{1}\right)$ : teniendo en cuenta que el centro de radiación de la energía debe encontrase por ejemplo a $1 / 3$ desde el límite inferior del foco, se considera que la extensión vertical del foco se define por la relación entre $h_{n} y h_{l}$ como se muestra en la ecuación 12, en la cual se despeja $h_{1} \mathrm{y}$ usando los valores de $h_{n} \mathrm{l}_{\mathrm{z}}$, calculados arriba, se obtiene que la profundidad local del foco sísmico es

$$
h_{l}=2,23 \mathrm{~km} \text {. }
$$

Este valor de profundidad, asociado a la culminación de la ruptura sísmica cerca de la superficie terrestre, se toma como la fuente de energía que ocasiona el efecto local, que explica la concentración de altos valores de intensidad en un área reducida, y las numerosas réplicas registradas por el evento, reportados en el análisis macrosísmico realizado por Sarabia y Cifuentes (2009), quienes también reportan la aparición de múltiples deslizamientos en la zona pleistosísmica de afectación del terremoto del 24 de mayo de 2008. 


\section{Aplicación de otras relaciones macrosísmicas empíricas}

Magnitud, energía liberada y momento sísmico. La magnitud y la energía de un terremoto dado pueden ser determinadas mediante relaciones empíricas si se conocen el radio de perceptibilidad de los efectos del sismo y su profundidad, usando las siguientes expresiones que son válidas para cualquier región (Gutenberg y Richter, 1942):

$$
\begin{gathered}
M_{L}=2,2+3,6 \log \frac{R}{h} \\
\log E=11,1+6,4 \log R-3,2 \log h
\end{gathered}
$$

Donde $\mathrm{M}_{\mathrm{L}}$ es la Magnitud de Richter; E es la Energía; $\mathrm{R}$, el radio de perceptibilidad, y $\mathrm{h}$ la profundidad focal. El límite de perceptibilidad del terremoto está definido como el área comprendida entre las isosistas de mayor valor y la de intensidad III (Bommer, 1994).

En este caso, el radio de perceptibilidad $\mathrm{R}$ se toma como la distancia al punto de intensidad III mejor determinado, con la certeza de la información macrosísmica recolectada. Según el mapa de intensidades propuesto por Sarabia y Cifuentes (2009), los puntos de intensidad III más confiables corresponden a los evaluados en los municipios Espinal y Melgar en el departamento del Tolima, ubicados a una distancia de 123 y 95 kilómetros, respectivamente, al suroccidente de la zona epicentral (ver Figura 3). De esta manera, el radio de perceptibilidad se toma como la distancia desde el epicentro al punto medio entre estas dos localidades, equivalente a 109 kilómetros. Cabe señalar que el mapa de intensidades elaborado por Sarabia y Cifuentes (2009) presenta otros valores inciertos de intensidad 3 localizados en dirección NW desde la zona de concentración de los mayores valores de intensidad, contrariando la distribución de las aceleraciones máximas obtenidas en la Red Nacional de Acelerógrafos que muestra un patrón de atenuación en dirección NE-SW, que además coincide con el mecanismo de falla propuesto por la Universidad de Harvard para este evento sísmico, y con la traza de la falla Naranjal a la cual se atribuye la ocurrencia del sismo (INGEOMINAS, 2008).

Bajo esta consideración, para el cálculo de la Magnitud de Richter $\left(\mathrm{M}_{\mathrm{L}}\right)$ y la Energía (E) se utiliza el radio de perceptibilidad y la profundidad normal $\mathrm{h}_{\mathrm{n}}$ determinados anteriormente. Con ello, usando las ecuaciones 19 y 20, se obtiene:

$$
\begin{gathered}
M_{L}=5,63 \\
E=4,57 * 10^{20} \text { ergios. }
\end{gathered}
$$

El momento sísmico escalar puede ser calculado en términos de la energía sísmica liberada a partir de la siguiente relación (Kanamori, 1977, 1983; Hanks y Kanamori, 1979; Abe, 1982; Bormann y di Giacomo, 2011):

$$
E=\frac{M_{0}}{2 * 10^{4}}
$$

Donde E es la energía sísmica liberada, dada en ergios, y $\mathrm{M}_{0}$ es el momento sísmico escalar.

La ecuación 21 fue propuesta por Kanamori (1977) para terremotos superficiales a partir de argumentos elasto-estáticos; sin embargo, Vassiliou y Kanamori (1982) sugieren que esta relación energía-momento también puede ser aplicada a terremotos profundos. De igual manera, Kanamori propuso la magnitud de momento Mw para eventos profundos y superficiales, a partir de $\mathrm{M}_{0}$, expresada como (Kanamori, 1977; Hanks y Kanamori, 1979; Kanamori, 1983):

$$
M w=\left(\log M_{0}-16,1\right) / 1,5
$$

Así, se obtiene que $\mathrm{M}_{0}=9,14 * 10^{24}$ dinas-cm y Mw = 5,9.

A partir de las intensidades consideradas en el mapa de isosistas, también es posible realizar un cálculo aproximado de la magnitud Ms considerando la siguiente ecuación (Bommer, 1994):

$$
M s=0,83 \log \left(\mathrm{r}_{3}^{2}\right)+0,28 I_{0}-0,13
$$

Ms - magnitud de ondas superficiales, $r_{3}$ - radio equivalente del límite de perceptibilidad (en kilómetros) e $\mathrm{I}_{0}$ - intensidad epicentral o la máxima intensidad registrada. Así, Ms=5,5.

\section{Resumen de parámetros focales}

Acorde con los métodos usados en el presente trabajo, se han obtenidos diversos parámetros que caracterizan las condiciones físicas del terremoto del 28 de mayo 2008. En la Tabla 3 se presenta un resumen de los parámetros focales (macrosísmicos) complementarios obtenidos en este estudio. 
Tabla 3. Parámetros focales complementarios del terremoto del 24 de mayo de 2008 en Quetame-Colombia, obtenidos a partir de datos macrosísmicos.

\begin{tabular}{lccc}
\hline \multicolumn{1}{c}{ Parámetro sismológico } & Símbolo & Unidad & Valor \\
\hline Epicentro macrosísmico normal & $E_{n}$ & grados & $\varphi=4,34^{\circ} \mathrm{N}$ \\
Profundidad normal del foco & $h_{n}$ & $\mathrm{~km}$ & $\lambda=73,86^{\circ} \mathrm{O}$ \\
Profundidad local del foco & $h_{l}$ & $\mathrm{~km}$ & 12,1 \\
Intensidad macrosísmica máxima & $I_{0}$ & escala EMS-98 & 2,23 \\
Magnitud de Richter & $M_{L}$ & adimensional & VIII $(8)^{\S}$ \\
Energía sísmica liberada & $E$ & ergios & 5,63 \\
Momento sísmico escalar & $M_{0}$ & dinas-cm & $4,57 * 10^{20}$ \\
Magnitud momento sísmico & $M w$ & adimensional & $9,14^{*} 10^{24}$ \\
Magnitud de ondas superficiales & $M s$ & adimensional & 5,9 \\
\hline
\end{tabular}

§Tomada de Sarabia y Cifuentes (2009)

\section{Deformación sismotectónica}

Para evaluar la deformación sismotectónica se aplica la complementariedad de los datos instrumentales y macrosísmicos, usando los datos del tensor de momento sísmico reportado por el GCMT, y los parámetros de dimensión del foco obtenidos en el presente estudio. El tensor de momento sísmico es expresado como:

$$
M_{i j}=\left[\begin{array}{rrr}
0,695 & -1,050 & 0,665 \\
-1,050 & 3,860 & 6,640 \\
0,665 & 6,640 & -4,560
\end{array}\right] * 10^{24} \text { dinas } \cdot \mathrm{cm}
$$

Ahora, puesto que el sismo en estudio es de profundidad superficial, el módulo de rigidez del medio puede considerarse como $\mu=3 \cdot 10^{11}$ dinas $\cdot \mathrm{cm}^{-2}$ (Turcotte y Schubert, 1982). De acuerdo con los datos reportados por la RSNC, se tiene que el proceso de réplicas asociado al terremoto del 24 de mayo de 2008, se prolongó hasta comienzos del mes de octubre de 2008; sin embargo, para efectos de considerar el tiempo $\mathrm{T}$, se tienen en cuenta sólo hasta el momento en que sus magnitudes inician un decrecimiento progresivo y conservan la localización epicentral alrededor del evento principal. En este caso, se tiene en cuenta el tiempo de registro de las principales réplicas del sismo hasta los primeros quince días desde la ocurrencia del sismo principal hasta el 7 de junio de 2008 (INGEOMINAS, 2008). Considerando el volumen de la fractura debido al proceso sísmico relacionado con este evento, se tiene que el tensor de deformación sísmica es:

$$
\dot{\varepsilon}_{i j}=\left[\begin{array}{rrr}
0,150 & -0,226 & 0,143 \\
-0,226 & 0,832 & 1,432 \\
0,143 & 1,432 & -0,983
\end{array}\right] * 10^{-9} a \tilde{n} o^{-1}
$$

Los valores propios del tensor de deformación son:

$$
\dot{\varepsilon}_{i j}=\left[\begin{array}{r}
-1,799 \\
0,167 \\
1,631
\end{array}\right] * 10^{-9} a \tilde{n} o^{-1}
$$

De esta forma, se tiene que la mayor tasa de deformación volumétrica producida en la región focal, ocurre en dirección N-S con magnitud aproximada de $1,80 * 10^{-9} \mathrm{año}^{-1}$ a una velocidad lineal, determinada por $\varepsilon x=\dot{\varepsilon}\left(l_{x}\right)$, de $0,041 \mathrm{~mm} / \mathrm{año}$. Este resultado es concordante con el movimiento producido por la falla Naranjal, con desplazamiento lateral izquierdo (INGEOMINAS, 2008; Cortés y De La Espriella, 1990). También se nota una importante tasa de deformación en la dirección hacia el centro de la Tierra, con magnitud $1,63 * 10^{-9} \mathrm{año}^{-1}$ con velocidad lineal de deformación, descrita por $\varepsilon_{z}=\dot{\varepsilon}\left(l_{z}\right)$, del orden de $0,024 \mathrm{~mm} / a n ̃ o$. Teniendo en cuenta que se trata de un sismo intraplaca de origen cortical, estos valores de velocidad de dislocación pueden considerarse como normales dentro del proceso de deformación gobernado por la actividad del fallamiento presente en la zona de ocurrencia del terremoto.

\section{Discusión}

Los datos macrosísmicos (intensidades) a diferencia de los instrumentales tienen la particularidad de que, a juicio de muchos, por su naturaleza pueden resultar dubitativos ya que son deducidos de los relatos, observaciones o cuestionarios que narran información, a veces dada por personas poco expertas, sobre los efectos y daños causados por un terremoto dado. Dicha información puede estar cargada de mucha 
subjetividad que debe ser interpretada por expertos quienes deben valorarla y encajarla a la luz de las escalas macrosísmicas existentes. Mientras que los datos instrumentales son deducidos de las formas de ondas registradas por instrumentos de los que es conocida la precisión y resolución del registro dadas por los respectivos fabricantes; pero también pueden contener algunas "impresiones" debidas al estado de conservación, calibración y la experticia de quienes leen e interpretan dichas formas de ondas, entre otros aspectos.

Además, como apunta Stucchi (1994), la diferencia real que tienen estos dos tipos de datos radica en que las formas de onda son más fáciles de obtener y los procedimientos mediante los cuales se procesan los datos instrumentales son conocidos y estandarizados, por lo cual los catálogos de datos instrumentales permiten ser comparados y evaluar su confiabilidad. En el caso de los datos macrosísmicos, éstos no se obtienen siguiendo procedimientos estandarizados, a veces son desconocidos, por lo que los registros de los catálogos correspondientes a diferentes conjuntos de datos y sus procedimientos no se pueden comparar fácilmente.

Sin embargo, a pesar del carácter subjetivo que guardan las asignaciones de los valores de intensidad, se puede decir que el uso de estos datos para interpretaciones sismológicos aplicando métodos macrosísmicos empíricos robustos, permite hacer una valoración apropiada de los parámetros focales de terremotos ocurridos en cualquier región geográfica, de tal manera que los estudios macrosísmicos pueden tomarse como una herramienta complementaria a los estudios que se desarrollan con datos instrumentales para la caracterización de terremotos históricos o recientes donde la cobertura y sensibilidad de las redes sismológicas sea baja.

La Red Sismológica Nacional de Colombia, usando trece estaciones sismológicas: Chingaza, Rosal, Tolima, Anillo, Refugio, Guaviare, Betania, Helena, Cóndor, Florencia, Ocaña, Montería y Cufiño, determinó el epicentro de este terremoto en las coordenadas geográficas $4,399^{\circ}$ latitud Norte y $73,814^{\circ}$ longitud Oeste, con profundidad superficial y magnitud local 5,7 (INGEOMINAS, 2008). Por su parte, la Universidad de Harvard, a través del proyecto GCMT, usando la red sismológica mundial, reportó el epicentro en las coordenadas $4,32^{\circ}$ latitud Norte y $73,79^{\circ}$ longitud Oeste, con profundidad de 14,7 km; además, estimaron magnitudes 5,9 Mw, 5,9 Ms, 5,6 mb y el momento sísmico escalar $\mathrm{M}_{0}=7,95 * 10^{24}$ dinas-cm.
Las diferencias presentadas en estas magnitudes, se atribuyen al hecho de que las escalas usadas consideran diferentes fases y tipos de registro de señal sísmica (INGEOMINAS, 2008). Se debe señalar que en estas diferencias también juega un papel muy importante la cobertura azimutal de las estaciones; el tipo, fidelidad y calibración de los equipos con los cuales se leen las fases de ondas para determinar los diversos parámetros focales. A pesar de ello, se nota coincidencia en las coordenadas epicentrales dadas por ambas agencias, más no en la profundidad, que la RSNC solo determina cómo superficial sin dar un valor preciso, que si es proporcionado por la Universidad de Harvard. Es importante resaltar que para la red sismológica mundial este evento es caracterizado como telesísmico, mientras que para la RSNC es de carácter local, por lo cual su comparación (Stucchi, 1994), permite mostrar sus diferencias.

Por esta razón, es importante obtener otro tipo de datos que, por su naturaleza y procesamiento, permitan aportar parámetros complementarios que den mejor conocimiento de este terremoto y sus datos puedan alimentar los catálogos sismológicos locales y regionales para futuros trabajos de evaluación de la amenaza y el riesgo sísmico. En este caso, los datos sismológicos complementarios son aportados por estudios macrosísmicos que, usando técnicas y métodos analíticos empíricos, también logran obtener parámetros focales similares y complementarios a los instrumentales. En este caso, estos parámetros se evalúan usando los datos macrosísmicos proporcionados en el mapa de intensidades propuesto por Sarabia y Cifuentes (2009). Los resultados que podrían ser comparados con los datos instrumentales, aunque por su naturaleza no tiene este fin (Stucchi, 1994), pero si deben tomarse como complementarios.

Se puede notar que entre las coordenadas epicentrales deducidas instrumental y macrosísmicamente no existen diferencias significativas. En cuanto a la profundidad focal, cabe señalar que desde el punto de vista macrosísmico, se hace diferencia entre la profundidad focal normal y local (Shebalin, 1974), puesto que la primera está relacionada con el volumen donde inicia la liberación de la energía del terremoto que es compatible con la dada instrumentalmente, mientras que la segunda se refiere al volumen donde terminaría la ruptura en el interior de la Tierra, causante de los efectos locales que produce el terremoto. Así, macrosísmicamente, se deduce que la profundidad normal es de $12,1 \mathrm{~km}$, valor muy 
similar al de 14,7 km obtenido instrumentalmente; mientras que la baja profundidad del foco local, con valor de 2,23 km, explica con mayor precisión la generación de los efectos locales y la severidad del sismo, manifestados en los deslizamientos y otros daños localizados en cercanías de la zona epicentral reportados por Sarabia y Cifuentes (2009). Esta profundidad no ha sido calculada en trabajos anteriores para este evento.

En torno a la magnitud, obsérvese que la Magnitud de Richter 5,7 $\mathrm{M}_{\mathrm{L}}$, reportada por la RSNC es muy similar a la obtenida macrosísmicamente $5,63 \mathrm{M}_{\mathrm{L}}$. La magnitud 5,9 Mw que se calcula por diferentes métodos macrosísmicos es igual a la reportada instrumentalmente por la Universidad de Harvard; cabe señalar que el método macrosísmico B\&W97, fija la profundidad de $10 \mathrm{~km}$, obligando a que geométricamente el centro de intensidades sea restringido a este hipocentro, posiblemente también restringe el cálculo de la magnitud. Igual pasa con la magnitud 5,6 mb, reportada instrumentalmente que corresponde al mismo valor obtenido macrosísmicamente. En la magnitud 5,9 Ms dada por la Universidad de Harvard, similar a la Mw, es un poco diferente a la obtenida macrosísmicamente 5,5 , esta diferencia puede ser explicada por el carácter lejano con que la red sismológica mundial analiza los datos instrumentales y el carácter local del incremento de la intensidad de los datos macrosísmicos. Por último, nótese que los valores del momento sísmico escalar obtenidos instrumental y macrosísmicamente son estrechamente similares, en los cuales sus exponentes son idénticos, mostrando una leve diferencia entre sus coeficientes. Adicionalmente, la energía sísmica evaluada en $4,57 * 10^{20}$ ergios, ha sido determinada mediante los datos macrosísmicos.

En la Tabla 4, se muestran la complementariedad de los diversos parámetros focales del terremoto del 24 de mayo de 2008, obtenidos mediante técnicas y métodos de análisis instrumentales y macrosísmicos.

Tabla 4. Parámetros focales del terremoto del 24 de mayo de 2008 en Quetame-Colombia, deducidos mediante técnicas y métodos instrumentales y macrosísmicos.

\begin{tabular}{|c|c|c|c|c|c|}
\hline \multirow{2}{*}{$\begin{array}{l}\text { Parámetro sismológico del terremoto } \\
\text { del } 24 \text { de mayo de } 2008 \text { obtenidos } \\
\text { mediante diferentes métodos } \\
\text { complementarios }\end{array}$} & \multicolumn{2}{|c|}{ Parámetros instrumentales } & \multicolumn{3}{|c|}{ Parámetros macrosísmicos } \\
\hline & GCMT & RSNC & $\begin{array}{c}\text { Bakun y } \\
\text { Wenworth }\end{array}$ & Shebalin & Otros \\
\hline \multirow{2}{*}{ Coordenadas Epicentro } & $\varphi=4,32^{\circ} \mathrm{N}$ & $\varphi=4,4^{\circ} \mathrm{N}$ & $\varphi=4,34^{\circ} \mathrm{N}$ & \multirow{2}{*}{--- } & \multirow{2}{*}{---} \\
\hline & $\lambda=73,79^{\circ} \mathrm{O}$ & $\lambda=73,81^{\circ} \mathrm{O}$ & $\lambda=73,86^{\circ} \mathrm{O}$ & & \\
\hline Profundidad normal del foco $\left(h_{n}\right)-k m$ & 14,7 & Superficial & 10 fijada & 12,1 & --- \\
\hline Profundidad local del foco $\left(\mathrm{h}_{1}\right)-\mathrm{km}$ & --- & --- & --- & 2,23 & --- \\
\hline Magnitud de Richter $\left(\mathrm{M}_{\mathrm{L}}\right)$ & --- & 5,7 & --- & --- & 5,63 \\
\hline Energía sísmica liberada (E) - ergios & --- & --- & --- & --- & $4,57 * 10^{20}$ \\
\hline Magnitud momento sísmico (Mw) & 5,9 & --- & 5,94 & --- & 5,9 \\
\hline Magnitud de ondas cuerpo (mb) & 5,6 & --- & $5,6 \pm 0,2$ & --- & --- \\
\hline Magnitud de ondas superficiales (Ms) & 5,9 & --- & --- & --- & 5,5 \\
\hline Momento sísmico escalar $\left(\mathrm{M}_{0}\right)-$ dinas $\cdot \mathrm{cm}$ & $7,95 * 10^{24}$ & --- & --- & --- & $9,14 * 10^{24}$ \\
\hline Tensor momento sísmico & $\begin{array}{c}* 10^{24} \\
\text { dinas-cm }\end{array}$ & & & & \\
\hline Mxx & 0,695 & & & & \\
\hline Mxy $=$ Myx & $-1,050$ & & & & \\
\hline$M x z=M z x$ & 0,665 & & & & \\
\hline Myy & 3,860 & & & & \\
\hline Myz $=$ Mzy & 6,640 & & & & \\
\hline Mzz & $-4,560$ & & & & \\
\hline Tensor deformación sísmica & & & & & *10-9 año-1 \\
\hline$\varepsilon_{\mathrm{xx}}$ & & & & & 0,150 \\
\hline$\varepsilon_{\mathrm{xy}}=\varepsilon_{\mathrm{yx}}$ & & & & & $-0,226$ \\
\hline$\varepsilon_{\mathrm{xz}}=\varepsilon_{\mathrm{zx}}$ & & & & & 0,143 \\
\hline$\varepsilon_{\mathrm{yy}}$ & & & & & 0,832 \\
\hline$\varepsilon_{\mathrm{yz}}=\varepsilon_{\mathrm{zy}}$ & & & & & 1,432 \\
\hline$\varepsilon_{\mathrm{yy}}$ & & & & & $-0,983$ \\
\hline
\end{tabular}




\section{Conclusión}

Se muestra el carácter complementario de los datos macrosísmicos e instrumentales de un terremoto reciente, sismo de Quetame, ocurrido el 24 de mayo de 2008, cuyos parámetros instrumentales han sido determinados por la RSNC y la Universidad de Harvard. Los efectos y daños causados en diversas poblaciones fueron evaluados por INGEOMINAS proponiendo hipótesis de intensidades en las escalas Mercalli Modificada y Escala Macrosísmica Europea (EMS-98). Estos últimos datos son utilizados en el presente trabajo para determinar parámetros focales (macrosísmicos) que complementan la información logrando el mejor conocimiento de las características físicas de este terremoto.

Usando los modelos macrosísmicos empíricos como los métodos B\&W97 y Shebalin, que a su vez aplican modelos de atenuación de la intensidad macrosísmica, se han determinado los parámetros focales del sismo de Quetame, arrojando datos totalmente compatibles con los reportados por las agencias sismológicas nacional e internacional como la RSNC y los publicados en los catálogos del ISC y del proyecto GCMT. Por ejemplo, las coordenadas del epicentro definidas instrumentalmente por GCMT y la RSNC corresponden a $4,32^{\circ} \mathrm{N}$ y $73,79^{\circ} \mathrm{O}$, y $4,4^{\circ} \mathrm{N}$ y $73,81^{\circ} \mathrm{O}$, respectivamente, mientras que macrosísmicamente se establecen en $4,34^{\circ} \mathrm{N}$ y $73,86^{\circ} \mathrm{O}$. Igualmente, se ha calculado la profundidad focal normal y local, donde la primera con valor de 12,1 kilómetros es compatible con la reportada instrumentalmente en 14,7 kilómetros, y la profundidad local en 2,23 km, la cual da cuenta de los efectos locales causados.

La aplicación de otras relaciones empíricas ha permitido la definición de la magnitud, cuyos valores obtenidos en diversas escalas $\left(M_{L}, m b, M s, M w\right)$, incluso el escalar momento sísmico $\mathrm{M}_{0}$, muestran gran similitud con los datos reportados instrumentalmente. En este caso, GCMT reporta $M_{0}=7,95 * 10^{24}$ dinas $\cdot \mathrm{cm}$, mientras que en este estudio se obtiene $M_{0}=9,14 * 10^{24}$.

El uso complementario de los datos instrumentales sobre las componentes del tensor de momento sísmico reportados por el GCMT de la Universidad de la Harvard y los parámetros de las dimensiones del foco sísmico obtenidos a partir del método Shebalin, permite determinar la tasa de deformación volumétrica o flujo sísmico de la masa rocosa, producida en la región focal, la cual ocurre con mayor preponderancia en la dirección N-S con magnitud aproximada de $1,80^{*} 10^{-9}$ año $0^{-1}$ a una velocidad lineal de deformación, determinada por la componente $\varepsilon_{x}=\varepsilon\left(l_{x}\right)$, correspondiente a $0,041 \mathrm{~mm} / \mathrm{año}$, valor concordante con el movimiento producido por la falla Naranjal, estructura tectónica a la cual la RSNC asoció la ocurrencia del evento.

Así, se puede asegurar que el uso de los métodos macrosísmicos robustos representa un camino alternativo y complementario para evaluar los parámetros focales de terremotos históricos o recientes permitiendo aportar información suficiente para alimentar los catálogos sismológicos nacional y local, útiles para el mejoramiento de los resultados de la amenaza y el riesgo sísmico en cualquier región geográfica sísmicamente activa, donde la información instrumental sea insuficiente.

\section{Agradecimientos}

Los autores agradecen al Observatorio Sismológico y Geofísico del Suroccidente Colombiano (OSSO) perteneciente a la Universidad del Valle, Colombia, a las directivas del Servicio Geológico Colombianos (antes INGEOMINAS) y Istituto Nazionale di Geofisica e Vulcanologia, sezione di Milano-Pavia por el apoyo y la colaboración para la realización de este trabajo. Expresamos sinceros agradecimientos a los revisores anónimos quienes, con sus comentarios y sugerencias, contribuyeron a mejorar la calidad y presentación de este trabajo.

\section{Referencias}

Abe, K. (1982). Magnitude, seismic moment and apparent stress for major deep earthquakes. Journal Physics Earth, 30(4), 321-330. https:// doi.org/10.4294/jpe1952.30.321

Aki, K.; Richards, P. (1980). Quantitative Seismology. Theory and Methods. Freeman.

Álvarez-Rubio, S.; Kästli, P.; Fäh, D.; Sellami, S.; Giardini, D. (2012). Parameterization of historical earthquakes in Switzerland. Journal of Seismology, 16(1), 1-24. https://doi.org/10.1007/ s10950-011-9245-8

Bakun, W.H. (2006). Estimating locations and magnitudes of earthquakes in Southern California from modified Mercalli intensities. Bulletin of the 
Seismological Society of America, 96(4A), 12781295. https://doi.org/10.1785/0120050205

Bakun, W.H.; Wentworth, C.M. (1997). Estimating earthquake location and magnitude from seismic intensity data. Bulletin of the Seismological Society of America, 87(6), 1502-1521.

Bakun, W.H.; Wentworth, C.M. (1999). Erratum to Estimating earthquake location and magnitude from seismic intensity data. Bulletin of the Seismological Society of America, 89(2), 557.

Bakun, W.H.; Gómez-Capera, A.; Stucchi, M. (2011). Epistemic uncertainty in the location and magnitude of earthquakes in Italy from macroseismic data. Bulletin of the Seismological Society of America, 101(6), 2712-2725. https:// doi.org/10.1785/0120110118

Bakun, W.H.; Flores, C.H.; Ten Brink, U.S. (2012). Significant Earthquakes on the Enriquillo Fault System, Hispaniola, 1500-2010: Implications for Seismic Hazard. Bulletin of the Seismological Society of America, 102(1), 18-30. https://doi. org/10.1785/0120110077

Beauval, C.; Yepes, H.; Bakun, W.H.; Egred, J.; Alvarado, A.; Singaucho, J.C. (2010). Locations and magnitudes of historical earthquakes in the Sierra of Ecuador (1587-1996). Geophysical Journal International, 181(3), 1613-1633. https:// doi.org/10.1111/j.1365-246X.2010.04569.x

Bindi, D.; Gómez-Capera, A.A.; Parolai, S.; Abdrakhmatov, K.; Stucchi, M.; Zschau, J. (2013). Location and magnitudes of earthquakes in Central Asia from seismic intensity data: model calibration and validation. Geophysical Journal International, 192(2), 710-724. https:// doi.org/10.1093/gji/ggs039

Bindi, D.; Parolai, S.; Gómez-Capera, A.; Locati, M.; Kalmetyeva, Z.; Mikhailova, N. (2014). Locations and magnitudes of earthquakes in Central Asia from seismic intensity data. Journal of Seismology, 18(1), 1-21. https://doi.org/10.1007/ s10950-013-9392-1

Blake, A. (1941). On the estimation of focal depth from macroseismic data. Bulletin of the Seismological Society of America, 31(3), 225-231.
Bolt, B.A. (2003). Earthquakes. 5th edition. W.H. Freeman \& Company.

Bommer, J. (1994). Sismología para ingenieros. Papeles Técnicos UCA. Serie Fundamentos F93001, Universidad Centroamericana José Simeón Cañas, San Salvador.

Bormann, P.; di Giacomo, D. (2011). The moment magnitude $M_{w}$ and the energy magnitude $M_{e}$ : common roots and differences. Journal of Seismology, 15(2), 411-427. https://doi. org/10.1007/s10950-010-9219-2

Bormann, P.; Baumbach, M.; Bock, G.; Grosser, H.; Choy, G.L.; Boatwright, J. (2002). Seismic sources and source parameters. In: P. Bormann (ed.). IASPEI New Manual of Seismological Observatory Practice (NMSOP). (pp. 1-94). Vol. 1. Ch 3. Deutsches GeoForschungszentrum GFZ; IASPEI.

Brune, J.N. (1968). Seismic moment, seismicity, and rate of slip along major fault zones. Journal of Geophysical Research, 73(2), 777-784. https:// doi.org/10.1029/JB073i002p00777

Brune, J.N. (1970). Tectonic stress and spectra of seismic shear waves from earthquakes. Journal of Geophysical Research, 75(26), 4997-5009.

Choy, J.E.; Palme, C.; Guada, C.; Morandi, M.; Klarica, S. (2010). Macroseismic interpretation of the 1812 earthquake in Venezuela using intensity uncertainties and a priori fault-strike information. Bulletin of the Seismological Society of America, 100(1), 241-255. https://doi. org/10.1785/0120080345

Cornell, C.A. (1986). Where do we go from here to increase the state of knowledge? Workshop on Probabilistic Earthquake-Hazards Assessments, San Francisco, California.

Cortés, R.; De La Espriella, R. (1990). Apuntes sobre la tectónica del valle del río Negro al Oriente de Cundinamarca. Geología Colombiana, 17, 133-142.

Global CMT Catalog. (2009). Catálogo CMT. Global Centroid Moment Tensor. http://www.globalcmt. org/ 
Gómez-Capera, A.A.; Salcedo-Hurtado, E. (2002). Leyes de atenuación de la intensidad macrosísmica en Colombia. Earth Sciences Research Journal, 6, 53-61.

Gómez-Capera, A.A.; Meletti, C.; Musson, R.; Stucchi, M. (2009). The European Earthquake Catalogue (1000-1600), demo version. Part 1-The NA4 Calibration Initiative, Deliverable D5. NERIES, Distributed Archive of Historical Earthquake Data (NA4), EC Project number 026130. Internal report.

Gómez-Capera, A.A.; García-Peláez, J.; ChuyRodríguez, T.; Salcedo-Hurtado, E.; Stucchi, M. (2013). Calibración y validación del algoritmo de Bakun and Wentworth para calcular localización y magnitud de terremotos históricos a partir de datos macrosísmicos en Cuba Oriental. Boletín de Geología, 35(2), 113-127.

Gómez-Capera, A.A.; Salcedo-Hurtado, E.; Bindi, D.; Choy, J.E.; García-Peláez, J. (2014). Localización y magnitud del terremoto de 1785 en Colombia calculadas a partir de intensidades macrosísmicas. Revista de la Academia Colombiana de Ciencias Exactas, Físicas y Naturales, 38(147), 206-217. https://doi.org/10.18257/raccefyn.63

Gómez-Capera, A.A.; Stucchi, M; Arcila, M.; Bufaliza, M.; Choy, J.; Leyton, L.; Pirchiner, M.; Rendon, H.; Rodriguez-Valverde, L.; Sarabia, A.M.; Tavera, H.; Yepes, H. (2017). Updated earthquake catalogue for South America: time window pre1964. 16th World Conference on Earthquake Engineering, Santiago de Chile, Chile.

Gómez-Capera, A.A.; Stucchi, M.; Arcila, M.; Bufaliza, M.; Choy J.; Leyton, L.; Pirchiner, M.; Rendon, H.; Rodriguez-Valverde, L.; Sarabia, A.M.; Tavera, H.; Yepes, H. (2020). Catálogo de terremotos de América del Sur homogéneo en Mw para el periodo pre-1964. GEOS, 40(1).

Grünthal, G. (2009). Escala Macrosísmica Europea 1998 EMS-98. Conseil de l'Europe, Cahiers du Centre Européen de Géodynamique et de Séismologie, Vol. 27. Edición española F. Lorenzo-Martín. Luxemburgo, Comisión Sismológica Europea.

Gutenberg, B.; Richter, C. (1942). Earthquake magnitude, intensity, energy and acceleration.
Bulletin of the Seismological Society of America, 32(3), 163-191.

Guzmán-Speziale, M. (2001). Active seismic deformation in the grabens of northern Central America and its relationship to the relative motion of the North America-Caribbean plate boundary. Tectonophysics, 337(1-2), 39-51. https://doi. org/10.1016/S0040-1951(01)00110-X

Hanks, T.C.; Kanamori, H. (1979). A moment magnitude scale. Journal of Geophysical Research, 84(B5), 2348-2350.

Haskell, N.A. (1964). Total energy and energy spectral density of elastic wave radiation from propagating faults. Bulletin of the Seismological Society of America, 54(6A), 1811-1841.

Heuret, A.; Lallemand, S.; Funiciello, F.; Piromallo, C.; Faccenna, C. (2011). Physical charac-teristics of subduction interface type seismogenic zones revisited. Geochemestry, Geophysics, Geosystems, 12(1). https://doi.org/10.1029/2010GC003230

Howell, B.F. Jr.; Schultz, T.R. (1975). Attenuation of Modified Mercalli intensity with distance from epicenter. Bulletin of the Seismological Society of America, 65(3), 651-665.

INGEOMINAS. (2008). El sismo de Quetame del 24 de mayo de 2008. Aspectos sismológicos y evaluación preliminar de daños. Informe Preliminar No. 2. INGEOMINAS, Subdirección de Amenazas Geológicas. Bogotá.

ISC. (2008). On-line Bulletin. International Seismological Centre.

Izquierdo-Álvarez, A. (1999). Intensidad Macrosísmica. Física de la Tierra, 11, 237-252.

Kanamori, H. (1977). The energy release in great earthquakes. Journal of Geophysical Research, 82(20), 2981-2987. https://doi.org/10.1029/ JB082i020p02981

Kanamori, H. (1983). Magnitude scale and quantification of earthquakes. Tectonophysics, 93(3-4), 185-199. https://doi.org/10.1016/00401951(83)90273-1 
Kanamori, H.; Astiz, L. (1985). The 1983 Akita-Oki Earthquake $\left(\mathrm{M}_{\mathrm{w}}=7.8\right)$ and its implications for systematic of subduction earthquakes. In: C. Kisslinger; T. Rikitake (ed.). Practical Approaches to Earthquake Prediction and Warning (pp. 305317). Springer. https://doi.org/10.1007/978-94017-2738-9_6

Karnik, V.; Algermissen, S.T. (1980). Zonificación sísmica. En: Terremotos, evaluación y mitigación de su peligrosidad (pp.11-49). UNESCO.

Kostrov, B. (1974). Mecánica del foco de terremotos tectónicos. Nauka. (en ruso).

Kostrov, B.; Das, S. (1988). Principals of Earthquakes Source Mechanics. Cambridge University Press.

Kövesligethy, R.D. (1906). A makroszeizmikus rengések feldolgozása. Mathematikai és Természettudományi Értesítõ, 24, 349-368.

Lay, T.; Wallace, T.C. (1995). Modern Global Seismology. Academic Press.

Madariaga, R. (1985). Dinámica de la fuente sísmica. Barreras y asperezas. En: A. Udías; D. Muñoz; E. Buforn (eds.). Mecanismo de los terremotos y tectónica (pp. 61-100). Editorial Complutense.

Madariaga, R. (1994). Dinámica de la fuente sísmica. Física de la Tierra, 6, 29-72.

Marín-Arias, J.P.; Castillo-González, H.; SalcedoHurtado, E. (2006). Modelo geométrico del foco del terremoto de Popayán (Colombia) a partir de datos macrosísmicos. Boletín de Geología, 28(2), 93-109.

Martín-Martín, A.J. (1989). Problemas relacionados con la evaluación de la peligrosidad sísmica en España. Física de la Tierra, 1, 267-286.

Mora, A.; Parra, M.; Strecker, M.R.; Kammer, A.; Dimaté, C.; Rodríguez, F. (2006). Cenozoic contractional reactivation of Mesozoic extensional structures in the Eastern Cordillera of Colombia. Tectonics, 25(2).

Mora-Páez, H.; López, S.; Acero, N; Ramírez-Cadena J.; Salcedo-Hurtado, E.; Trenkamp, R. (2015). Análisis geodésico y deformación sismotectónica asociada al sismo de Quetame, Colombia,
24 de mayo de 2008. Boletín Geológico, 43, 7-22. https://doi.org/10.32685/0120-1425/ boletingeo.43.2015.26

Mulargia, F.; Stark, P.B.; Geller, R.J. (2017). Why is Probabilistic Seismic Hazard Analysis (PSHA) still used? Physics of the Earth and Planetary Interiors, 264, 63-75. https://doi.org/10.1016/j. pepi.2016.12.002

Patiño, A.; Fuquen, J.; Ramos, J.; Pedraza, A.; Ceballos, L.; Pinzón, L.; Jerónimo, Y.; Álvarez, L.; Torres, A. (2011). Geología de la Plancha 247 Bogotá Sur Este (Cáqueza) escala 1:100.000. INGEOMINAS.

Reiter, L. (1990). Earthquake hazard analysis: issues and insights. Columbia University Press.

Riznichenko, Y.V. (1965). Relación entre el flujo sísmico de la masa rocosa y la sismicidad. Informes de la Academia de Ciencias de la URSS, 161(1), 97-99. (en ruso).

Riznichenko, Y.V. (1976). Dimensiones del foco de sismos corticales y momento sísmico. En: C.A. Fedotov (ed.). Investigaciones sobre física de los terremotos (pp. 9-27). Naúka. (en ruso).

Riznichenko, Y.V.; Dzibladze, E.A. (1976). Velocidad de los movimientos verticales causados por flujo sísmico de la masa rocosa. Revista Física de la Tierra, 1, 23-31. (en ruso).

Salcedo-Hurtado, E. (2002). Sismicidad histórica y análisis macrosísmico de Bucaramanga. Boletín Geológico, 40(1), 1-180.

Salcedo-Hurtado, E.; Castaño-Castaño, A.N. (2011). Reevaluación macrosísmica del terremoto del 12 de julio de 1785 en Colombia. Boletín de Geología, 33(2), 15-32.

Salcedo-Hurtado, E.; Gómez-Capera, A.A. (2013). Estudio macrosísmico del terremoto del 18 octubre del 1743 en la región central de Colombia. Boletín de Geología, 35(1), 109-128.

Sarabia, A.M.; Cifuentes, H.G. (2009). Análisis macrosísmico del sismo del 24 de mayo de 2008 en Quetame. Reporte Interno INGEOMINAS, Bogotá. 
Savage, J.C. (1966). Radiation from a Realistic model of faulting. Bulletin of the Seismological Society of America, 56(2), 577-592.

Shebalin, N.V. (1968). Problemas de la naturaleza de los sismos profundos. Informes de la Academia de Ciencias de la URSS, 181(5) 1119-1122. (en ruso).

Shebalin, N.V. (1971). Cálculo de las dimensiones y posición del foco del sismo de Tashkien mediante datos macrosísmicos e instrumentales. En: T.N. Kallaur (ed.). El sismo de Tashkien de 1966 (pp. 68-79). Academia de Ciencias de Uzbekistán. (en ruso).

Shebalin, N.V. (1974). Focos de sismos fuertes en el territorio de la URSS. Nauka. (en ruso).

Shebalin, N.V. (1991). Cálculo del tamaño y posición del foco del terremoto de Spitak mediante datos macrosísmicos. En: N.I. Frolova, C.D. Vinogradov (eds.). Problemas de la Ingeniería Sísmica (pp. 35-44). No. 32. Naúka. (en ruso).

Stein, S.; Wysession, M. (2003). An introduction to seismology, earthquakes, and structure. Blackwell Publishing.

Stepp, J.C. (1972). Analysis of completeness of the earthquake sample in the Puget Sound area and its effect on statistical estimates of earthquake hazard. International Conference on Microzonation, Seattle, USA.

Storchak, D.A.; di Giacomo, D.; Bondár, I.; Harris, J.; Engdahl, E.R.; Lee, W.H.K.; Villaseñor, A.; Bormann, P.; Ferrari, G. (2012). ISC-GEM Global Instrumental Earthquake Catalogue (1900-2009). GEM Technical Report.
Storchak, D.A.; di Giacomo, D.; Bondár, I.; Engdahl, E.R.; Harris, J.; Lee, W.H.K.; Villaseñor, A.; Bormann, P. (2013). Public release of the ISC-GEM Global Instrumental Earthquake Catalogue (19002009). Seismological Research Letters, 84(5), 810-815. https://doi.org/10.1785/0220130034

Stucchi, M. (1994). Recommendations for the compilation of a European parametric earthquake catalogue, with special reference to historical records. In: P. Albini; A. Moroni (ed.). Materials of the CEC Project Review of Historical Seismicity in Europe (pp. 181-190). Vol 2. CNR.

Turcotte, D.L.; Schubert, G. (1982). Geodynamics. Applications of continuum physics to geological problems. John Wiley and Sons.

Udías, A. (1994). Mecanismo de los terremotos: modelos cinemáticos. Física de la Tierra, 6, 1127.

Udías, A.; Mezcua, J. (1997). Fundamentos de geofísica. Alianza Editorial. 1ra ed.

Vassiliou, M.S.; Kanamori, H. (1982). The energy release in earthquakes. Bulletin of the Seismological Society of America, 72(2), 371387.

Zsìros, T. (1995). Macroseismics focal depth and intensity attenuation in the Carpathian Region. Acta Geodaetica et Geophysica Hungarica, 31(1), 115-125.

Fecha de recibido: 25 de julio de 2020

Fecha de aprobado: 11 de marzo de 2021 